\title{
HOMOTOPY TYPES OF GAUGE GROUPS OVER NON-SIMPLY-CONNECTED CLOSED 4-MANIFOLDS
}

\author{
TSELEUNG SO
}

\begin{abstract}
Let $G$ be a simple, simply-connected, compact Lie group and let $M$ be an orientable, smooth, connected, closed 4-manifold. In this paper we calculate the homotopy type of the suspension of $M$ and the homotopy types of the gauge groups of principal $G$ bundles over $M$ when $\pi_{1}(M)$ is (1) $\mathbb{Z}^{* m},(2) \mathbb{Z} / p^{r} \mathbb{Z}$, or $(3) \mathbb{Z}^{* m} *\left(*_{j=1}^{n} \mathbb{Z} / p_{j}^{r_{j}} \mathbb{Z}\right)$, where $p$ and the $p_{j}$ 's are odd primes.
\end{abstract}

\section{INTRODUCTION}

Let $G$ be a topological group and let $M$ be a topological space. Given a principal $G$ bundle $P$ over $M$, the associated gauge group $\mathcal{G}(P)$ is the topological group of $G$-equivariant automorphisms of $P$ which fix $M$. Atiyah, Bott and Gottlieb [1, 4] showed that its classifying space $B \mathcal{G}(P)$ is homotopy equivalent to the connected component $\operatorname{Map}_{P}(M, B G)$ of the mapping space $\operatorname{Map}(M, B G)$ which contains the map inducing $P$. When $G$ is a simple, simply-connected, compact Lie group and $M$ is an orientable, smooth, connected, closed 4manifold, it can be shown that the set of isomorphism classes of principal $G$-bundles over $M$ is in one-to-one correspondence with the homotopy set $[M, B G] \cong \mathbb{Z}$. If a principal $G$-bundle corresponds to an integer $t$, then we denote its associated gauge group by $\mathcal{G}_{t}(M)$. In [18] Theriault shows that when $M$ is a simply-connected spin 4-manifold, there is a homotopy equivalence

$$
\mathcal{G}_{t}(M) \simeq \mathcal{G}_{t}\left(S^{4}\right) \times \prod_{i=1}^{n} \Omega^{2} G,
$$

where $n$ is the rank of $H^{2}(M ; \mathbb{Z})$. For the non-spin case this homotopy equivalence still holds when localized away from 2 . As a result, the study of $\mathcal{G}_{t}(M)$ can be reduced to that of $\mathcal{G}_{t}\left(S^{4}\right)$, which has been being investigated over the last twenty years. Kishimoto, Kono and Tsutaya gave bounds on the numbers of distinct homotopy types of $\mathcal{G}_{t}\left(S^{4}\right)$ for all $G$ using localization at odd primes [9]. Moreover, the homotopy types of $\mathcal{G}_{t}\left(S^{4}\right)$ are classified for many cases. Let $(a, b)$ be the greatest common divisor of $a$ and $b$. Then $[3,8,10,11,19,21,22]$

- when $G=S U(2)$, there is a homotopy equivalence $\mathcal{G}_{t}\left(S^{4}\right) \simeq \mathcal{G}_{s}\left(S^{4}\right)$ if and only if $(12, t)=(12, s)$;

- when $G=S U(3)$, there is a homotopy equivalence $\mathcal{G}_{t}\left(S^{4}\right) \simeq \mathcal{G}_{s}\left(S^{4}\right)$ if and only if $(24, t)=(24, s)$;

- when $G=S U(5)$, there is a $p$-homotopy equivalence $\mathcal{G}_{t}\left(S^{4}\right) \simeq \mathcal{G}_{s}\left(S^{4}\right)$ if and only if $(120, t)=(120, s)$ for any prime $p$;

2010 Mathematics Subject Classification. Primary 54C35, 55P40; Secondary 55R10.

Key words and phrases. homotopy decomposition, gauge groups, 4-manifolds. 
- when $G=S p(2)$, there is a $p$-local homotopy equivalence $\mathcal{G}_{t}\left(S^{4}\right) \simeq \mathcal{G}_{s}\left(S^{4}\right)$ if and only if $(40, t)=(40, s)$ for any prime $p$;

- when $G=S U(n)$, there is a $p$-local homotopy equivalence $\mathcal{G}_{t}\left(S^{4}\right) \simeq \mathcal{G}_{s}\left(S^{4}\right)$ if and only if $\left(n\left(n^{2}-1\right), t\right)=\left(n\left(n^{2}-1\right), s\right)$ for any odd prime $p$ such that $n \leq(p-1)^{2}+1$;

- when $G=S p(n)$, there is a $p$-local homotopy equivalence $\mathcal{G}_{t}\left(S^{4}\right) \simeq \mathcal{G}_{s}\left(S^{4}\right)$ if and only if $(4 n(2 n+1), t)=(4 n(2 n+1), s)$ for any odd prime $p$ such that $2 n \leq(p-1)^{2}+1$;

- when $G=G_{2}$, there is a $p$-local homotopy equivalence $\mathcal{G}_{t}\left(S^{4}\right) \simeq \mathcal{G}_{s}\left(S^{4}\right)$ if and only if $(84, t)=(84, s)$ for any odd prime $p$,

In addition, a few cases of $\mathcal{G}_{t}\left(\mathbb{C P}^{2}\right)$ are worked out $[12,20]$ :

- when $G=S U(2)$, there is a homotopy equivalence $\mathcal{G}_{t}\left(\mathbb{C P}^{2}\right) \simeq \mathcal{G}_{s}\left(\mathbb{C P}^{2}\right)$ if and only if $(6, t)=(6, s)$;

- when $G=S U(3)$, there is a $p$-local homotopy equivalence $\mathcal{G}_{t}\left(\mathbb{C P}^{2}\right) \simeq \mathcal{G}_{s}\left(\mathbb{C P}^{2}\right)$ if and only if $(12, t)=(12, s)$ for any prime $p$.

On the other hand, very little is known about $\mathcal{G}_{t}(M)$ when $M$ is non-simply-connected. The goal of this paper is to study the homotopy types of $\mathcal{G}_{t}(M)$ for certain non-simplyconnected 4-manifolds. To achieve this we need a homotopy decomposition statement.

Theorem 1.1. Suppose that $G$ is a simple, simply-connected, compact Lie group and $Y$ is a CW-complex of dimension at most 3. Let $\phi: Y \rightarrow M$ be a map such that $\Sigma \phi$ has a left homotopy inverse. Then we have

$$
\mathcal{G}_{t}(M) \simeq \mathcal{G}_{t}\left(C_{\phi}\right) \times \operatorname{Map}^{*}(Y, G),
$$

where $C_{\phi}$ is the cofiber of $\phi$.

Using Theorem 1.1 we calculate the homotopy types of $\mathcal{G}_{t}(M)$ when $\pi_{1}(M)$ is (1) $\mathbb{Z}^{* m}$, (2) $\mathbb{Z} / p^{r} \mathbb{Z}$, or $(3) \mathbb{Z}^{* m} *\left(*_{j=1}^{n} \mathbb{Z} / p_{j}^{r_{j}} \mathbb{Z}\right)$, where $p$ and the $p_{j}$ 's are odd primes.

Theorem 1.2. Suppose that $G$ is a simple, simply-connected, compact Lie group and $M$ is an orientable, smooth, connected, closed 4-manifold.

- If $\pi_{1}(M)=\mathbb{Z}^{* m}$ or $\mathbb{Z} / p^{r} \mathbb{Z}$, then $\mathcal{G}_{t}(M)$ is homotopy equivalent to a product of $\mathcal{G}_{t}\left(S^{4}\right)$ or $\mathcal{G}_{t}\left(\mathbb{C P}^{2}\right)$ and "loop spaces" on $G$.

- If $\pi_{1}(M)=\mathbb{Z}^{* m} *\left(*_{j=1}^{n} \mathbb{Z} / p_{j}^{r_{j}} \mathbb{Z}\right)$, then $\mathcal{G}_{t}(M) \times \prod^{2 d} \Omega^{2} G$ is homotopy equivalent to a product of $\mathcal{G}_{t}\left(S^{4}\right)$ or $\mathcal{G}_{t}\left(\mathbb{C P}^{2}\right)$ and "loop spaces" on $G$ for some number $d$.

The term "loop spaces" refers both to iterated based loop spaces $\Omega G, \Omega^{2} G$ and $\Omega^{3} G$ and modular loop spaces $\Omega G\left\{p^{r}\right\}$ and $\Omega^{2} G\left\{p^{r}\right\}$, where $G\left\{p^{r}\right\}$ is the homotopy fiber of the $p^{r}$ power map on $G$. Explicit decompositions are stated in Section 3.

Theorem 1.2 shows that the homotopy type of $\mathcal{G}_{t}(M)$ is related to that of $\mathcal{G}_{t}\left(S^{4}\right)$ or $\mathcal{G}_{t}\left(\mathbb{C P}^{2}\right)$ in these three cases. Combining Theorem 1.2 and the known results in [3, 11, 10, 19, 22], we have the following classification.

Corollary 1.3. If $M$ is an orientable, smooth, connected, closed 4-manifold with $\pi_{1}(M)=\mathbb{Z}^{* m}$ or $\mathbb{Z} / p^{r} \mathbb{Z}$, then the followings hold:

- when $G=S U(2)$, there is a homotopy equivalence $\mathcal{G}_{t}(M) \simeq \mathcal{G}_{s}(M)$ if and only if $(12, t)=(12, s)$ for $M$ spin, and $(6, t)=(6, s)$ for $M$ non-spin; 
- when $G=S U(3)$, there is a homotopy equivalence $\mathcal{G}_{t}(M) \simeq \mathcal{G}_{s}(M)$ if and only if $(24, t)=(24, s)$ for $M$ spin; there is a p-local homotopy equivalence $\mathcal{G}_{t}(M) \simeq \mathcal{G}_{s}(M)$ if and only if $(12, t)=(12, s)$ for any prime $p$ and $M$ non-spin;

- when $G=S U(n)$, there is a p-local homotopy equivalence $\mathcal{G}_{t}(M) \simeq \mathcal{G}_{s}(M)$ if and only if $\left(n\left(n^{2}-1\right), t\right)=\left(n\left(n^{2}-1\right), s\right)$ for any odd prime $p$ such that $n \leq(p-1)^{2}+1$;

- when $G=S p(2)$, there is a p-local homotopy equivalence $\mathcal{G}_{t}(M) \simeq \mathcal{G}_{s}(M)$ if and only if $(40, t)=(40, s)$ for any odd prime $p$;

- when $G=G_{2}$, there is a p-local homotopy equivalence $\mathcal{G}_{t}(M) \simeq \mathcal{G}_{s}(M)$ if and only if $(84, t)=(84, s)$ for any odd prime $p$.

There is an analogous statement to Corollary 1.3.

Corollary 1.4. If $M$ is an orientable, smooth, connected, closed 4-manifold with $\pi_{1}(M)=$ $\mathbb{Z}^{* m} *\left(*_{j=1}^{n} \mathbb{Z} / p_{j}^{r_{j}} \mathbb{Z}\right)$, then the integral and $p$-local homotopy equivalences in Corollary 1.3 hold for $\mathcal{G}_{t}(M) \times \prod^{2 d} \Omega^{2} G$ for some number $d$.

The structure of this paper is as follows. In Section 2 we prove the homotopy decomposition Theorem 1.1 and develop some useful lemmas. In particular, Theorem 1.1 is used to revise homotopy equivalence (1) which is often referred to during the calculations in Section 3 . In Section 3 we give the homotopy types of $\Sigma M$ and $\mathcal{G}_{t}(M)$ when $\pi_{1}(M)$ is either $\mathbb{Z}^{* m}, \mathbb{Z} / p^{r} \mathbb{Z}$ or $\mathbb{Z}^{* m} *\left(*_{j=1}^{n} \mathbb{Z} / p_{j}^{r_{j}} \mathbb{Z}\right)$, where $p$ and the $p_{j}$ 's are odd primes.

The author would like to thank the reviewer for carefully reading the paper and giving many constructive comments.

\section{A homotopy Decomposition of Gauge Groups}

2.1. A homotopy decomposition and some useful lemmas. We are going to extend the homotopy decomposition (1) to a more general situation. Suppose that $M$ is an orientable 4-dimensional CW-complex that is constructed by attaching a 4-cell onto a 3-dimensional CW-complex $M_{3}$ by an attaching map $f: S^{3} \rightarrow M_{3}$. Let $u: B G \rightarrow K(\mathbb{Z}, 4)$ be a generator of $H^{4}(B G) \cong \mathbb{Z}$. Since it is a 5 -equivalence, $u_{*}:[M, B G] \rightarrow[M, K(\mathbb{Z}, 4)]=\mathbb{Z}$ is a bijection. If a principal $G$-bundle over $M$ corresponds to some integer $t$, then we denote its associated gauge group by $\mathcal{G}_{t}(M)$.

In [18], the homotopy decomposition (1) is obtained as a consequence of the attaching map $f$ of the 4-cell in $M$ having the property that $\Sigma f$ is null-homotopic. Observe that $\Sigma f$ is the connecting map of the cofibration sequence

$$
M_{3} \longrightarrow M \longrightarrow S^{4}
$$

where $M_{3}$ is the 3 -skeleton of $M$. From the point of view of homotopy theory, it can be replaced by a cofibration sequence

$$
Y \stackrel{\phi}{\longrightarrow} M \stackrel{q}{\longrightarrow} C_{\phi}
$$

for some space $Y$ and some map $\phi: Y \rightarrow M$, with a connecting map that is null-homotopic. Here $q$ is the quotient map and $C_{\phi}$ is the cofiber of $\phi$. The nullity condition is equivalent to $\Sigma \phi$ having a left homotopy inverse. If we further restrict the dimension of $Y$ to be at most 3, then by Cellular Approximation Theorem $\phi$ is homotopic to $\imath \circ \varphi$, where $\varphi: Y \rightarrow M_{3}$ 
is a map on $M_{3}$ and $\imath: M_{3} \rightarrow M$ is the inclusion. The existence of a left homotopy inverse of $\Sigma \phi$ imposes a strong condition on $\Sigma M$.

Lemma 2.1. Let $Y$ be a $C W$-complex of dimension at most 3 and let $\phi: Y \rightarrow M$ be a map. If $\phi$ is homotopic to $\imath \circ \varphi$ for some $\operatorname{map} \varphi: Y \rightarrow M_{3}$, then the following are equivalent:

(1) $\Sigma \phi: \Sigma Y \rightarrow \Sigma M$ has a left homotopy inverse $\psi$;

(2) $\Sigma \varphi$ has a left homotopy inverse $\psi^{\prime}$ and $\psi^{\prime} \circ \Sigma f$ is null-homotopic;

(3) $\Sigma M$ is homotopy equivalent to $\Sigma Y \vee \Sigma C_{\phi}$ and $\Sigma \phi$ is homotopic to the inclusion;

(4) $\Sigma M_{3}$ is homotopy equivalent to $\Sigma Y \vee \Sigma C_{\varphi}$ where $C_{\varphi}$ is the cofiber of $\varphi, \Sigma \varphi$ is homotopic to the inclusion and $\psi^{\prime} \circ \Sigma f$ is null-homotopic.

Proof. First we show that Condition (1) and (2) are equivalent. If $\Sigma \phi$ has a left homotopy inverse $\psi$, then $\psi^{\prime}=\psi \circ \Sigma \imath$ is a left homotopy inverse of $\Sigma \varphi$ and $\psi^{\prime} \circ \Sigma f=\psi \circ \Sigma \imath \circ \Sigma f$ is null-homotopic since $S^{4} \stackrel{\Sigma f}{\longrightarrow} \Sigma M_{3} \stackrel{\Sigma \imath}{\longrightarrow} \Sigma M$ is a cofibration sequence. Conversely, assume Condition (2). Consider the homotopy commutative diagram

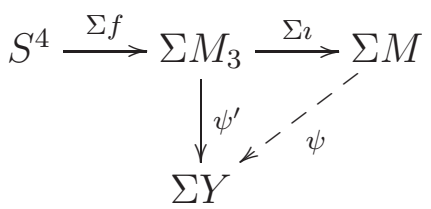

By hypothesis $\psi^{\prime} \circ \Sigma f$ is null-homotopic, so $\psi^{\prime}$ has an extension $\psi$. Then we have

$$
\begin{aligned}
\psi \circ \Sigma \phi & \simeq \psi \circ \Sigma(\imath \circ \varphi) \\
& \simeq(\psi \circ \Sigma \imath) \circ \Sigma \varphi \\
& \simeq \psi^{\prime} \circ \Sigma \varphi \\
& \simeq \mathbb{1}_{\Sigma Y},
\end{aligned}
$$

where $\mathbb{1}_{\Sigma Y}$ is the identity map on $\Sigma Y$. Therefore $\psi$ is a left homotopy inverse of $\Sigma \phi$.

Now we show that Conditions (1) and (3) are equivalent. If $\Sigma \phi$ has a left homotopy inverse $\psi$, then let $h$ be the composition

$$
h: \Sigma M \stackrel{\sigma}{\longrightarrow} \Sigma M \vee \Sigma M \stackrel{\psi \vee \Sigma q}{\longrightarrow} \Sigma Y \vee \Sigma C_{\phi},
$$

where $\sigma$ is the comultiplication. Observe that $h$ induces an isomorphism $H^{*}\left(\Sigma Y \vee \Sigma C_{\phi}\right) \rightarrow$ $H^{*}(\Sigma M)$. Since these spaces are suspensions and are simply-connected, $h$ is a homotopy equivalence by Whitehead Theorem. Therefore $\Sigma M$ is homotopy equivalent to $\Sigma Y \vee \Sigma C_{\phi}$. Moreover, since $\Sigma \phi$ is a co-H-map,

$$
\begin{aligned}
h \circ \Sigma \phi & \simeq(\psi \vee \Sigma q) \circ \sigma \circ \Sigma \phi \\
& \simeq(\psi \vee \Sigma q) \circ(\Sigma \phi \vee \Sigma \phi) \circ \sigma \\
& \simeq(\psi \circ \Sigma \phi \vee \Sigma q \circ \Sigma \phi) \circ \sigma \\
& \simeq\left(\mathbb{1}_{\Sigma Y} \vee *\right) \circ \sigma
\end{aligned}
$$

is the inclusion $\Sigma Y \hookrightarrow \Sigma Y \vee \Sigma C_{\phi}$. Conversely, assume Condition (3). Let $h$ be a homotopy equivalence from $\Sigma M$ to $\Sigma Y \vee \Sigma C_{\phi}$ and let $\psi$ be the composition

$$
\psi: \Sigma M \stackrel{h}{\longrightarrow} \Sigma Y \vee \Sigma C_{\phi} \stackrel{p}{\longrightarrow} \Sigma Y \text {. }
$$


where $p$ is the pinch map. By hypothesis, $h \circ \Sigma \phi$ is homotopic to the inclusion $\Sigma Y \rightarrow \Sigma Y \vee \Sigma C_{\phi}$, so we have

$$
\psi \circ \Sigma \phi \simeq p \circ h \circ \Sigma \phi \simeq \mathbb{1}_{\Sigma Y}
$$

and $\psi$ is a left homotopy inverse of $\Sigma \phi$.

The equivalence between (2) and (4) can be shown similarly.

We can extend the cofibration (2) to the homotopy commutative diagram whose rows and columns are cofibration sequences

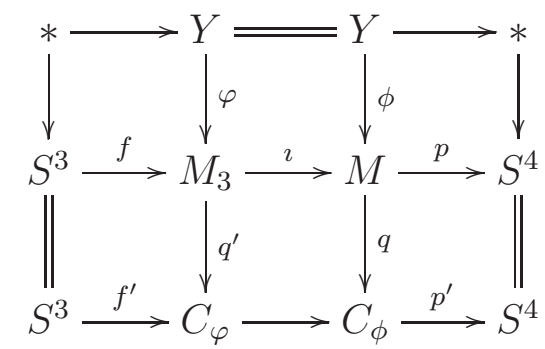

where $q^{\prime}, p$ and $p^{\prime}$ are the quotient maps and $f^{\prime}=q^{\prime} \circ f$. The bottom row implies that $C_{\phi}$ is constructed by attaching a 4-cell onto $C_{\varphi}$ via $f^{\prime}$. The generator $u$ of $H^{4}(B G)$ induces a bijection between $\left[C_{\phi}, B G\right]$ and $\left[C_{\phi}, K(\mathbb{Z}, 4)\right] \cong \mathbb{Z}$, so any principal $G$-bundle over $C_{\phi}$ corresponds to some integer $t$. Denote the associated gauge group by $\mathcal{G}_{t}\left(C_{\phi}\right)$. We want to compare $\mathcal{G}_{t}(M)$ and $\mathcal{G}_{t}\left(C_{\phi}\right)$ via the pullback $q^{*}$.

Lemma 2.2. Assume the conditions in Lemma 2.1. Then $q^{*}:\left[C_{\phi}, B G\right] \rightarrow[M, B G]$ is a group isomorphism.

Proof. The naturality of $u^{*}$ implies the commutative diagram

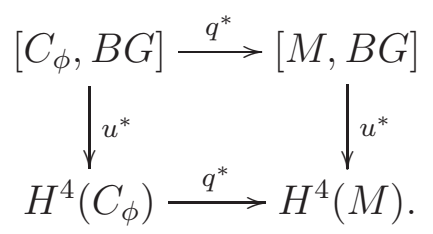

Since the group structures on $[M, B G]$ and $\left[C_{\phi}, B G\right]$ are induced by bijections

$$
u^{*}:[M, B G] \rightarrow H^{4}(M) \cong \mathbb{Z} \text { and } u^{*}:\left[C_{\phi}, B G\right] \rightarrow H^{4}\left(C_{\phi}\right) \cong \mathbb{Z},
$$

it suffices to show that $q^{*}: H^{4}\left(C_{\phi}\right) \rightarrow H^{4}(M)$ is an isomorphism.

The cofibration (2) induces an exact sequence

$$
H^{4}(\Sigma M) \stackrel{(\Sigma \phi)^{*}}{\longrightarrow} H^{4}(\Sigma Y) \stackrel{\epsilon^{*}}{\longrightarrow} H^{4}\left(C_{\phi}\right) \stackrel{q^{*}}{\longrightarrow} H^{4}(M) \stackrel{\phi^{*}}{\longrightarrow} H^{4}(Y)
$$

where $\epsilon^{*}$ is induced by the connecting map $\epsilon: C_{\phi} \rightarrow \Sigma Y$. Since $\Sigma \phi$ has a left homotopy inverse, $\epsilon$ is null-homotopic and $\epsilon^{*}$ is trivial. Also, the dimension of $Y$ is at most 3, so $H^{4}(Y)$ is trivial. By exactness $q^{*}: H^{4}\left(C_{\phi}\right) \rightarrow H^{4}(M)$ is an isomorphism.

Lemma 2.3. For any integer $t$, let $M a p_{t}^{*}(M, B G)$ and $M a p_{t}^{*}\left(C_{\phi}, B G\right)$ be the connected components of $\operatorname{Map}^{*}(M, B G)$ and $\operatorname{Map}^{*}\left(C_{\phi}, B G\right)$ containing $t \in[M, B G] \cong\left[C_{\phi}, B G\right]$ respectively. Then

$$
\operatorname{Map}^{*}(\Sigma Y, B G) \stackrel{\epsilon^{*}}{\longrightarrow} \operatorname{Map}_{t}^{*}\left(C_{\phi}, B G\right) \stackrel{q^{*}}{\longrightarrow} \operatorname{Map}_{t}^{*}(M, B G)
$$

is a homotopy fibration sequence. 
Proof. First, $q^{*}: \operatorname{Map}_{t}^{*}\left(C_{\phi}, B G\right) \rightarrow \operatorname{Map}_{t}^{*}(M, B G)$ is well defined by Lemma 2.2. Second, the cofibration (2) induces a homotopy fibration sequence

$$
\operatorname{Map}^{*}(\Sigma Y, B G) \stackrel{\epsilon^{*}}{\longrightarrow} \operatorname{Map}^{*}(M, B G) \stackrel{q^{*}}{\longrightarrow} \operatorname{Map}^{*}\left(C_{\phi}, B G\right)
$$

Since $\epsilon^{*}$ is null-homotopic, $\operatorname{Map}^{*}(\Sigma Y, B G)$ is mapped into $\operatorname{Map}_{0}^{*}(M, B G)$ and hence is the homotopy fiber of $q^{*}: \operatorname{Map}_{0}^{*}(M, B G) \rightarrow \operatorname{Map}_{0}^{*}\left(C_{\phi}, B G\right)$. To show that it is true for any $t \in \mathbb{Z}$, let $\alpha_{t}: \operatorname{Map}_{0}^{*}(M, B G) \rightarrow \operatorname{Map}_{t}^{*}(M, B G)$ be a map sending a pointed map $g: M \rightarrow B G$ to the composition

$$
\alpha_{t}(g): M \stackrel{c}{\longrightarrow} M \vee S^{4} \stackrel{g \vee t}{\longrightarrow} B G \vee B G \stackrel{\nabla}{\longrightarrow} B G,
$$

where $c$ is the coaction map of $M$ and $\nabla$ is the folding map, and let $\beta_{t}: \operatorname{Map}_{0}^{*}\left(C_{\phi}, B G\right) \rightarrow$ $\operatorname{Map}_{t}^{*}\left(C_{\phi}, B G\right)$ be a map defined similarly. Since $\alpha_{t}$ and $\beta_{t}$ are homotopy equivalences, it suffices to prove the commutate diagram

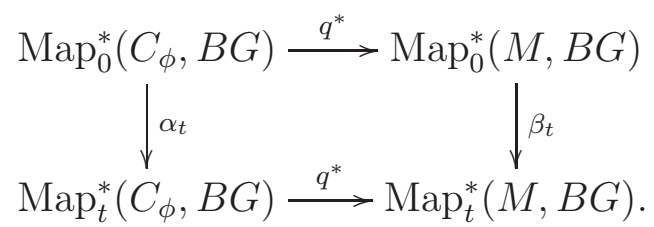

Consider the homotopy commutative diagram

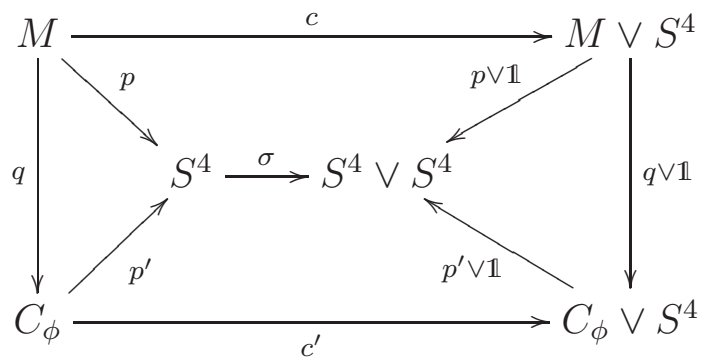

where $c^{\prime}$ is the coaction map, and $p$ and $p^{\prime}$ are the quotient maps, and $\sigma$ is the comultiplication of $S^{4}$. The left and the right triangles are due to the bottom right square in diagram (3), the top and the bottom quadrangles are due to the property of coaction maps. Extend it to get the cofibration diagram

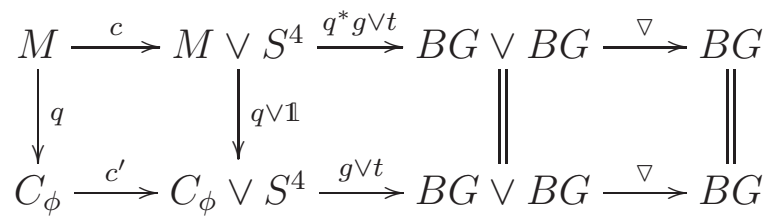

The upper row around the diagram is $\beta_{t}\left(q^{*} g\right)$, while the lower row around the diagram is $q^{*} \alpha_{t}(g)$. Therefore $q^{*}$ commutes with $\alpha_{t}$ and $\beta_{t}$ and the asserted statement follows.

Theorem 2.4. Let $Y$ be a $C W$-complex of dimension at most 3 and let $\phi: Y \rightarrow M$ be a map. If $\phi$ satisfies one of the four conditions in Lemma 2.1, then there are homotopy equivalences

$$
\Sigma M \simeq \Sigma C_{\phi} \vee \Sigma Y \text { and } \mathcal{G}_{t}(M) \simeq \mathcal{G}_{t}\left(C_{\phi}\right) \times \operatorname{Map}^{*}(Y, G) .
$$

Proof. Let $\operatorname{Map}_{t}(M, B G)$ and $\operatorname{Map}_{t}\left(C_{\phi}, B G\right)$ be the connected components of $\operatorname{Map}(M, B G)$ and $\operatorname{Map}\left(C_{\phi}, B G\right)$ containing $t \in[M, B G] \cong\left[C_{\phi}, B G\right]$. There are evaluation fibrations

$$
\operatorname{Map}_{t}^{*}(M, B G) \rightarrow \operatorname{Map}_{t}(M, B G) \rightarrow B G \text { and } \operatorname{Map}_{t}^{*}\left(C_{\phi}, B G\right) \rightarrow \operatorname{Map}_{t}\left(C_{\phi}, B G\right) \rightarrow B G \text {. }
$$


By Lemma $2.2 q^{*}:\left[C_{\phi}, B G\right] \rightarrow[M, B G]$ is an isomorphism, so there is a homotopy commutative diagram whose rows are fibration sequences

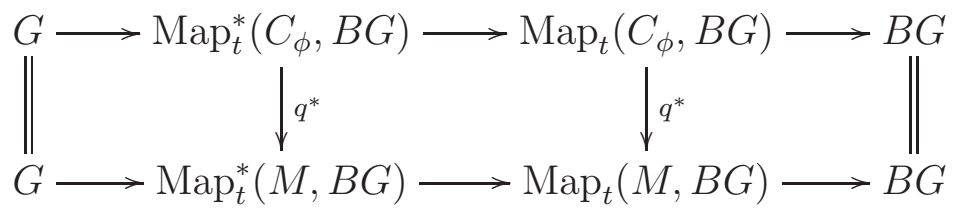

As in [18], from the left square in (4) we obtain a homotopy fibration diagram

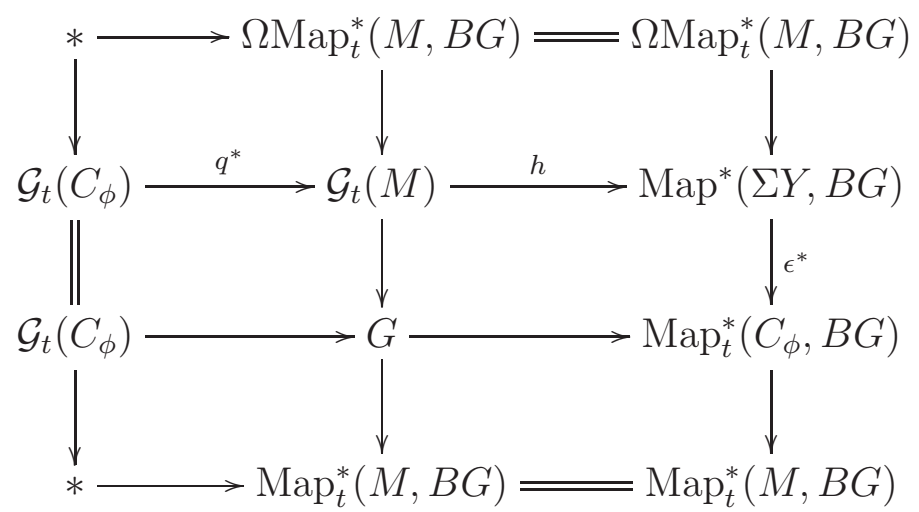

The right column is due to Lemma 2.3. The nullity of $\epsilon^{*}$ implies that $h$ has a right homotopy inverse. The group multiplication in $\mathcal{G}_{t}(M)$ then gives a homotopy equivalence

$$
\Phi: \mathcal{G}_{t}\left(C_{\phi}\right) \times \operatorname{Map}^{*}(\Sigma Y, B G) \longrightarrow \mathcal{G}_{t}(M) \times \mathcal{G}_{t}(M) \longrightarrow \mathcal{G}_{t}(M),
$$

so $\mathcal{G}_{t}(M)$ is homotopy equivalent to $\mathcal{G}_{t}\left(C_{\phi}\right) \times \operatorname{Map}^{*}(Y, G)$.

When calculating $\mathcal{G}_{t}(M)$, we will use Theorem 2.4 several times and apply it to $M$ and $C_{\phi}$ in some cases. So here we establish some facts about $C_{\phi}$.

Lemma 2.5. Let $q^{\prime}: M \rightarrow C_{\phi}$ be the quotient map and let $f^{\prime}$ be the composition

$$
S^{3} \stackrel{f}{\longrightarrow} M_{3} \stackrel{q^{\prime}}{\longrightarrow} C_{\varphi} .
$$

Then $\Sigma f^{\prime}$ is null-homotopic if and only if $\Sigma f$ is null-homotopic.

Proof. The necessity is obvious. Assume that $\Sigma f^{\prime}$ is null-homotopic. Since the composition

$$
h: \Sigma M_{3} \stackrel{\sigma}{\longrightarrow} \Sigma M_{3} \vee \Sigma M_{3} \stackrel{\psi \vee \Sigma q^{\prime}}{\longrightarrow} \Sigma Y \vee \Sigma C_{\varphi}
$$

is a homotopy equivalence, it suffices to show that $h \circ \Sigma f$ is null-homotopic. Consider the homotopy commutative diagram

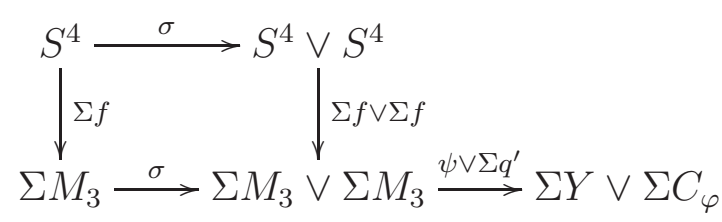

where the two $\sigma$ 's are the comultiplications of $S^{4}$ and $\Sigma M_{3}$. The lower direction around the diagram is $h \circ \Sigma f$, and the upper direction around the diagram is

$$
\left(\psi \circ \Sigma f \vee \Sigma q^{\prime} \circ \Sigma f\right) \circ \sigma \simeq\left(\psi \circ \Sigma f \vee \Sigma f^{\prime}\right) \circ \sigma
$$


By hypothesis $\Sigma f^{\prime}$ is null-homotopic, and by Lemma 2.1(4) $\psi \circ \Sigma f$ is null-homotopic. Therefore $h \circ \Sigma f$ is null-homotopic and hence so is $\Sigma f$.

Lemma 2.6. Let $H_{\text {free }}^{2}(X)$ be the free part of $H^{2}(X)$ for any space $X$. If $H^{2}(Y)$ is torsion, then $q^{*}: H_{\text {free }}^{2}\left(C_{\phi}\right) \rightarrow H_{\text {free }}^{2}(M)$ is an isomorphism. Moreover, if for any $\alpha^{\prime} \in H_{\text {free }}^{2}(M)$ there exists $\beta^{\prime} \in H_{\text {free }}^{2}(M)$ such that $\alpha^{\prime} \cup \beta^{\prime} \in H^{4}(M)$ is a generator, then for any $\alpha \in H_{\text {free }}^{2}\left(C_{\phi}\right)$ there exists $\beta \in H_{\text {free }}^{2}\left(C_{\phi}\right)$ such that $\alpha \cup \beta \in H^{4}\left(C_{\phi}\right)$ is a generator.

Proof. Cofibration (2) induces the long exact sequence of cohomology groups

$$
\cdots \longrightarrow H^{k}\left(C_{\phi}\right) \stackrel{(\Sigma q)^{*}}{\longrightarrow} H^{k}(M) \stackrel{(\Sigma \phi)^{*}}{\longrightarrow} H^{k}(Y) \longrightarrow \cdots
$$

Since $\Sigma \phi$ has a left homotopy inverse, the sequence splits for $k \geq 1$ and we have

$$
H^{k}(M) \cong H^{k}(Y) \oplus H^{k}\left(C_{\phi}\right)
$$

By hypothesis $H^{2}(Y)$ is torsion, so $H_{\text {free }}^{2}\left(C_{\phi}\right)$ is isomorphic to $H_{\text {free }}^{2}(M)$.

For any $\alpha \in H_{\text {free }}^{2}\left(C_{\phi}\right), q^{*}(\alpha)$ is in $H_{\text {free }}^{2}(M)$. By hypothesis there exists $\beta^{\prime} \in H_{\text {free }}^{2}(M)$ such that $q^{*}(\alpha) \cup \beta^{\prime} \in H^{4}(M)$ is a generator. Since $q^{*}: H_{\text {free }}^{2}\left(C_{\phi}\right) \rightarrow H_{\text {free }}^{2}(M)$ is an isomorphism, there exists $\beta \in H_{\text {free }}^{2}\left(C_{\phi}\right)$ such that $q^{*}(\beta)=\beta^{\prime}$. Therefore we have $q^{*}(\alpha) \cup \beta^{\prime}=q^{*}(\alpha \cup \beta)$. Observe that $q^{*}: H^{4}\left(C_{\phi}\right) \rightarrow H^{4}(M)$ is an isomorphism since $Y$ has dimension at most 3 . It follows that $\alpha \cup \beta$ is a generator of $H^{4}\left(C_{\phi}\right)$.

The second part of Lemma 2.6 says that the cup product on $H_{\text {free }}^{2}\left(C_{\phi}\right)$ is unimodular if the cup product on $H_{\text {free }(M)}^{2}$ is unimodular, which follows from Poincaré Duality when $M$ is an orientable compact manifold. Furthermore, if $C_{\phi}$ has a subcomplex $Y^{\prime}$ satisfying Theorem 2.4, then the cup product on $H_{\text {free }}^{2}\left(C_{\phi} / Y^{\prime}\right)$ is still unimodular.

Next we consider two variations of Theorem 2.4 when $M$ has a special structure.

Lemma 2.7. Suppose that $M_{3}$ is homotopy equivalent to $Z \vee Z^{\prime}$. Let $Y$ and $Y^{\prime}$ be $C W$ complexes of dimension at most 3, and let $\varphi: Y \rightarrow Z$ and $\varphi^{\prime}: Y^{\prime} \rightarrow Z^{\prime}$ be maps. If $\Sigma \varphi$ and $\Sigma \varphi^{\prime}$ have left homotopy inverses $\psi$ and $\psi^{\prime}$ respectively and the compositions

$$
S^{4} \stackrel{\Sigma f}{\longrightarrow} \Sigma M_{3} \stackrel{\text { pinch }}{\longrightarrow} \Sigma Z \stackrel{\psi}{\longrightarrow} \Sigma Y \quad \text { and } \quad S^{4} \stackrel{\Sigma f}{\longrightarrow} \Sigma M_{3} \stackrel{\text { pinch }}{\longrightarrow} \Sigma Z^{\prime} \stackrel{\psi^{\prime}}{\longrightarrow} \Sigma Y^{\prime},
$$

are null-homotopic, then we have

$$
\Sigma M \simeq \Sigma M^{\prime} \vee \Sigma Y \vee \Sigma Y^{\prime} \text { and } \mathcal{G}_{t}(M) \simeq \mathcal{G}_{t}\left(M^{\prime}\right) \times \operatorname{Map}^{*}(\Sigma Y, B G) \times \operatorname{Map}^{*}\left(\Sigma Y^{\prime}, B G\right)
$$

where $M^{\prime}$ is the cofiber of the map $Y \vee Y^{\prime} \stackrel{\varphi \vee \varphi^{\prime}}{\longrightarrow} Z \vee Z^{\prime} \hookrightarrow M$.

Proof. Let $\Phi$ be the composition

$$
\Phi: Y \vee Y^{\prime} \stackrel{\varphi \vee \varphi^{\prime}}{\longrightarrow} Z \vee Z^{\prime} \simeq M_{3}
$$

The map $\psi \vee \psi^{\prime}: \Sigma Z \vee \Sigma Z^{\prime} \rightarrow \Sigma Y \vee \Sigma Y^{\prime}$ is a left homotopy inverse of $\Sigma \Phi$. We show that $\left(\psi \vee \psi^{\prime}\right) \circ \Sigma f$ is null-homotopic, implying that $\Phi$ satisfies the hypothesis of Theorem 2.4.

Notice that the composition

$$
h: \Sigma M_{3} \stackrel{\sigma}{\longrightarrow} \Sigma M_{3} \vee \Sigma M_{3} \stackrel{p_{1} \vee p_{2}}{\longrightarrow} \Sigma Z \vee \Sigma Z^{\prime}
$$


is a homotopy equivalence, where $p_{1}: \Sigma M_{3} \rightarrow \Sigma Z$ and $p_{2}: \Sigma M_{3} \rightarrow \Sigma Z^{\prime}$ are the pinch maps. Since $\Sigma f$ is a co-H-map, we have

$$
\begin{aligned}
\left(\psi \vee \psi^{\prime}\right) \circ h \circ \Sigma f & \simeq\left(\psi \vee \psi^{\prime}\right) \circ\left(p_{1} \vee p_{2}\right) \circ \sigma \circ \Sigma f \\
& \simeq\left(\psi \vee \psi^{\prime}\right) \circ\left(p_{1} \vee p_{2}\right) \circ(\Sigma f \vee \Sigma f) \circ \sigma \\
& \simeq\left(\psi \circ p_{1} \circ \Sigma f \vee \psi^{\prime} \circ p_{2} \circ \Sigma f\right) \circ \sigma
\end{aligned}
$$

which is null-homotopic by assumption. Therefore $\left(\psi \vee \psi^{\prime}\right) \circ \Sigma f$ is null-homotopic and Theorem 2.4 implies the asserted statement.

Lemma 2.8. Suppose $M \cong X \# X^{\prime}$ where $X$ and $X^{\prime}$ are orientable, smooth, connected, closed 4-manifolds. Let $Y$ and $Y^{\prime}$ be $C W$-complexes of dimensions at most 3 and let $\varphi: Y \rightarrow X_{3}$ and $\varphi^{\prime}: Y^{\prime} \rightarrow X_{3}^{\prime}$ be maps satisfying the hypothesis of Theorem 2.4. Then we have

$$
\Sigma M \simeq \Sigma M^{\prime} \vee \Sigma Y \vee \Sigma Y^{\prime} \text { and } \mathcal{G}_{t}(M) \simeq \mathcal{G}_{t}\left(M^{\prime}\right) \times \operatorname{Map}^{*}(\Sigma Y, B G) \times \operatorname{Map}^{*}\left(\Sigma Y^{\prime}, B G\right)
$$

where $M^{\prime}$ is the cofiber of the inclusion $Y \vee Y^{\prime} \stackrel{\varphi \vee \varphi^{\prime}}{\longrightarrow} X_{3} \vee X_{3}^{\prime} \hookrightarrow M$.

Proof. Let $f: S^{3} \rightarrow X_{3}$ and $f^{\prime}: S^{3} \rightarrow X_{3}^{\prime}$ be the attaching maps of the 4-cells in $X$ and $X^{\prime}$ respectively. By Lemma 2.1, $\Sigma \varphi$ and $\Sigma \varphi^{\prime}$ have left homotopy inverses $\psi$ and $\psi^{\prime}$ and $\psi \circ \Sigma f$ and $\psi^{\prime} \circ \Sigma f^{\prime}$ are null-homotopic. We show that $\varphi$ and $\varphi^{\prime}$ satisfy the hypothesis in Lemma 2.7.

The 3-skeleton of $M$ is $X_{3} \vee X_{3}^{\prime}$ and the attaching map of the 4-cell is

$$
f_{\#}: S^{3} \stackrel{\sigma}{\longrightarrow} S^{3} \vee S^{3} \stackrel{f \vee f^{\prime}}{\longrightarrow} X_{3} \vee X_{3}^{\prime}
$$

Let $p_{1}: \Sigma X_{3} \vee \Sigma X_{3}^{\prime} \rightarrow \Sigma X_{3}$ and $p_{2}: \Sigma X_{3} \vee \Sigma X_{3}^{\prime} \rightarrow \Sigma X_{3}^{\prime}$ be the pinch maps. Consider the homotopy commutative diagram

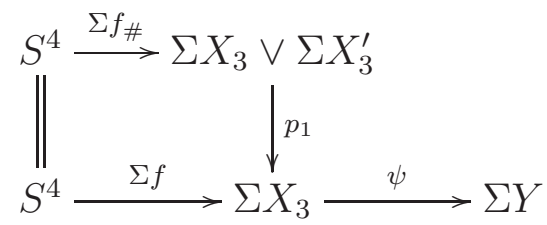

Since $\psi \circ \Sigma f$ is null-homotopic, the composition $\psi \circ p_{1} \circ \Sigma f_{\#}$ is null-homotopic. Similarly $\psi^{\prime} \circ p_{2} \circ \Sigma f_{\#}$ is null-homotopic. Then Lemma 2.7 implies the statement.

2.2. Gauge groups over simply-connected 4-manifolds. Now we revise homotopy equivalence (1) using Theorem 2.4. When $M$ is simply-connected, its 3-skeleton $M_{3}$ is homotopy equivalent to $\bigvee_{i=1}^{n} S^{2}$. If $\Sigma f$ is null-homotopic, then we can apply Theorem 2.4 by taking $Y$ to be the whole of $M_{3}$ and $\varphi: Y \rightarrow M_{3}$ to be the identity map and get the homotopy equivalence (1). In the following we assume the homotopy class of $\Sigma f$ is not trivial.

To distinguish the 2 -spheres, denote the $i^{\text {th }}$ copy of $S^{2}$ in $M_{3}$ by $S_{i}^{2}$. Let $\eta: S^{3} \rightarrow S^{2}$ be the Hopf map and let $\eta_{i}$ be the composition

$$
\eta_{i}: S^{3} \stackrel{\eta}{\longrightarrow} S_{i}^{2} \hookrightarrow \bigvee_{i=1}^{n} S_{i}^{2} .
$$

We also denote the suspensions $\Sigma \eta$ and $\Sigma \eta_{i}$ by $\bar{\eta}$ and $\bar{\eta}_{i}$ for short.

Lemma 2.9. If $M_{3}$ is homotopy equivalent to $\bigvee_{i=1}^{n} S_{i}^{2}$, then $\Sigma f$ is homotopic to $\sum_{i=1}^{n} a_{i} \bar{\eta}_{i}$ where $a_{i} \in \mathbb{Z} / 2 \mathbb{Z}$. 
Proof. The Hilton-Milnor Theorem implies

$$
\pi_{3}\left(\bigvee_{i=n}^{n} S_{i}^{2}\right) \cong \bigoplus_{i=1}^{n} \pi_{3}\left(S_{i}^{2}\right) \oplus \bigoplus_{i \neq j} \pi_{3}\left(\Sigma S_{i}^{1} \wedge S_{j}^{1}\right),
$$

where $\pi_{3}\left(S_{i}^{2}\right)$ is generated by $\eta_{i}$ and $\pi_{3}\left(\Sigma S_{i}^{1} \wedge S_{j}^{1}\right)$ is generated by Whitehead products of the identity maps on $S_{i}^{2}$ and $S_{j}^{2}$. As an element in $\pi_{3}\left(\bigvee_{i=1}^{n} S_{i}^{2}\right), f$ is homotopic to $\sum_{i=1}^{n} \tilde{a}_{i} \eta_{i}+w$ where $\tilde{a}_{i}$ is an integer and $w$ is a sum of Whitehead products in $\bigoplus_{i \neq j} \pi_{3}\left(S_{i}^{2} \wedge S_{j}^{1}\right)$. After suspension $\Sigma f$ is homotopic to $\sum_{i=1}^{n} a_{i} \bar{\eta}_{i}$, where $a_{i} \equiv \tilde{a}_{i}(\bmod 2)$, since suspensions of Whitehead products are null-homotopic and $\bar{\eta}_{i}$ has order 2 .

If the homotopy class of $\Sigma f$ is not trivial, then at least one of the $a_{i}$ 's is not zero. Relabelling the spheres if necessary, we may assume that $\Sigma f$ is homotopic to $\sum_{i=1}^{m} \bar{\eta}_{i}$ for some integer $m$ such that $1 \leq m \leq n$. Then we can simplify this expression with the following lemma.

Lemma 2.10. If $M_{3}$ is homotopy equivalent to $\bigvee_{i=1}^{n} S_{i}^{2}$ and $\Sigma f$ is homotopic to $\sum_{i=1}^{m} \bar{\eta}_{i}$, then there is a map $\tilde{f}: S^{3} \rightarrow M_{3}$ such that its cofiber $C_{\tilde{f}}$ is homotopy equivalent to $M$ and $\Sigma \tilde{f}$ is homotopic to $\bar{\eta}_{1}$. Moreover, $p_{1} \circ \tilde{f}$ is homotopic to $p_{1} \circ f$ where $p_{1}: \bigvee_{i=1}^{n} S_{i}^{2} \rightarrow S_{1}^{2}$ is the pinch map.

Proof. For each $1<j \leq m$, define a map $\xi_{j}: M_{3} \rightarrow M_{3}$ as follows. On $S_{1}^{2} \vee S_{j}^{2}, \xi_{j}$ is the composition

$$
S_{1}^{2} \vee S_{j}^{2} \stackrel{\sigma \vee \mathbb{1}}{\longrightarrow} S_{1}^{2} \vee S_{1}^{2} \vee S_{j}^{2} \stackrel{\mathbb{1} \vee \nabla}{\longrightarrow} S_{1}^{2} \vee S_{j}^{2},
$$

where $\sigma$ is a comultiplication of $S_{1}^{2}$ and $\nabla$ is the folding map of $S_{1}^{2}$ and $S_{j}^{2}$. On the remaining spheres, $\xi_{j}$ is the identity. Consider the homotopy cofibration diagram

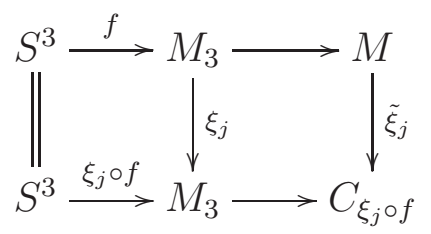

where $\tilde{\xi}_{j}$ is an induced map and $C_{\xi_{j} \circ f}$ is the cofiber of $\xi_{j} \circ f$. Since $\xi_{j}$ is a homology isomorphism, so is $\tilde{\xi}_{j}$ by the 5-lemma. Therefore $\tilde{\xi}_{j}$ is a homotopy equivalence and $M$ is homotopy equivalent to $C_{\xi_{j} \circ f}$.

By Lemma $2.9, \Sigma\left(\xi_{j} \circ f\right)$ is homotopic to $\sum_{i=1}^{n} a_{i} \bar{\eta}_{i}$, where $a_{i} \in \mathbb{Z} / 2 \mathbb{Z}$. For $1 \leq i \leq n$, let $p_{i}: \bigvee_{i=1}^{n} S_{i}^{3} \rightarrow S_{i}^{3}$ be the pinch map. Then $a_{i}$ is $p_{i} \circ \Sigma\left(\xi_{j} \circ f\right)$. By the definition of $\xi_{j}$, $a_{i}=1$ for $i \neq j$ and $1 \leq i \leq m$, and $a_{i}=0$ otherwise, that is

$$
\Sigma\left(\xi_{j} \circ f\right) \simeq \bar{\eta}_{1}+\cdots+\bar{\eta}_{j-1}+\bar{\eta}_{j+1}+\cdots+\bar{\eta}_{m}
$$

Let $\tilde{f}$ be $\xi_{m} \circ \cdots \circ \xi_{2} \circ f$ and let $C_{\tilde{f}}$ be its cofiber. Then $\Sigma \tilde{f}$ is homotopic to $\bar{\eta}_{1}$ and $C_{\tilde{f}}$ is homotopy equivalent to $M$.

Lastly, observe that $p_{1} \circ \xi_{j}$ is homotopic to $p_{1}$. It follows that $p_{1} \circ \xi_{j} \circ f$ is homotopic to $p_{1} \circ f$ and so is $p_{1} \circ \tilde{f}$. 
Lemma 2.11. Let $M$ be a 4-dimensional simply-connected $C W$-complex. If $\Sigma f$ is nontrivial, then there are homotopy equivalences

$$
\Sigma M \simeq \Sigma \mathbb{C P}^{2} \vee\left(\bigvee_{i=1}^{n-1} S^{3}\right) \text { and } \mathcal{G}_{t}(M) \simeq \mathcal{G}_{t}\left(C_{a \eta}\right) \times \prod_{i=1}^{n-1} \Omega^{2} G,
$$

where $a$ is an odd integer, $n$ is the rank of $H^{2}(M)$ and $C_{a \eta}$ is the cofiber of an.

Proof. By Lemma $2.9 \Sigma f$ is homotopic to $\sum_{i=1}^{m} \bar{\eta}_{i}$ for some $m$ such that $1 \leq m \leq n$. By Lemma 2.10 there exists a map $\tilde{f}$ such that $\Sigma \tilde{f}$ is homotopic to $\bar{\eta}_{1}$ and its cofiber $\tilde{M}$ is homotopy equivalent to $M$. Replacing $f$ by $\tilde{f}$ and $M$ by $\tilde{M}$, we can assume $\Sigma f \simeq \bar{\eta}_{i}$. Use Theorem 2.4 by taking $Y$ to be $\bigvee_{i=2}^{n} S_{i}^{2}$ and $\phi: \bigvee_{i=2}^{n} S_{i}^{2} \rightarrow M$ to be the inclusion and get

$$
\Sigma M \simeq \Sigma C_{\phi} \vee\left(\bigvee_{i=1}^{n-1} S^{3}\right) \text { and } \mathcal{G}_{t}(M) \simeq \mathcal{G}_{t}\left(C_{\phi}\right) \times \prod_{i=1}^{n-1} \Omega^{2} G,
$$

where $C_{\phi}$ is the cofiber of $\phi$. We need to show that $C_{\phi}$ is homotopy equivalent to $C_{a \eta}$ for some odd integer $a$ and $\Sigma C_{\phi}$ is homotopy equivalent to $\Sigma \mathbb{C P}^{2}$.

Consider the cofibration diagram

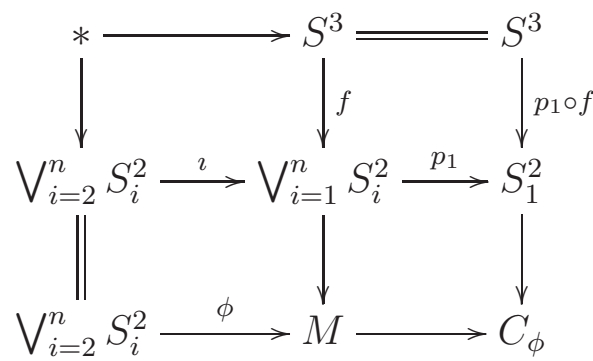

where $\imath: \bigvee_{i=2}^{n} S_{i}^{2} \rightarrow \bigvee_{i=1}^{n} S_{i}^{2}$ is the inclusion, $p_{1}: \bigvee_{i=2}^{n} S_{i}^{3} \rightarrow S_{1}^{2}$ is the pinch map. Since $p_{1} \circ f$ is in $\pi_{3}\left(S^{2}\right) \cong \mathbb{Z}$, it is homotopic to a $\eta$ for some integer $a$. The right column implies that $C_{\phi}$ is homotopy equivalent to the cofiber $C_{a \eta}$ of $a \eta$. Moreover, $\Sigma f$ is homotopic to $\bar{\eta}_{1}$, so $\Sigma\left(p_{1} \circ f\right)$ is homotopic to $\Sigma \eta$ and $a$ is an odd number. It follows that $\Sigma C_{\phi}$ is homotopy equivalent to the cofiber of $\Sigma \eta$, which is $\Sigma \mathbb{C P}^{2}$.

We can modify the result of Lemma 2.11 a bit better.

Lemma 2.12. Let a be an odd number. Then we have

$$
\mathcal{G}_{t}\left(C_{a \eta}\right) \times \Omega^{2} G \simeq \mathcal{G}_{t}\left(\mathbb{C P}^{2}\right) \times \Omega^{2} G
$$

Proof. Let $g$ be the composition

$$
g: S^{3} \stackrel{\sigma}{\longrightarrow} S^{3} \vee S^{3} \stackrel{\eta \vee a \eta}{\longrightarrow} S^{2} \vee S^{2}
$$

and let $C_{g}$ be its cofiber. To distinguish the 2-spheres in the range, denote the $i^{\text {th }}$ copy by $S_{i}^{2}$. Now we calculate $\mathcal{G}_{t}\left(C_{g}\right)$. By Lemma 2.10, we can assume that $\Sigma g$ is homotopic to $\bar{\eta}_{1}$ and $p_{1} \circ g$ is homotopic to $\eta$. Use Theorem 2.4 by taking $Y$ to be $S_{2}^{2}$ and $\varphi: S_{2}^{2} \rightarrow S_{1}^{2} \vee S_{2}^{2}$ to be the inclusion and obtain $\mathcal{G}_{t}\left(C_{g}\right) \simeq \mathcal{G}_{t}\left(C_{g} / S_{2}^{2}\right) \times \Omega^{2} G$. Consider the homotopy cofibration 
diagram

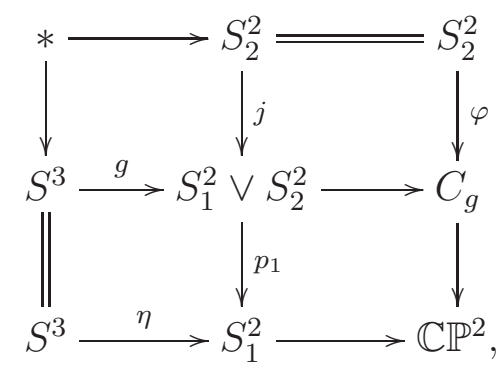

where $j$ is the inclusion and $p_{1}$ is the pinch map. The right column implies that $C_{g} / S_{2}^{2}$ is homotopy equivalent to $\mathbb{C P}^{2}$, so we have

$$
\mathcal{G}_{t}\left(C_{g}\right) \simeq \mathcal{G}_{t}\left(\mathbb{C P}^{2}\right) \times \Omega^{2} G
$$

Similarly, let $g^{\prime}$ be composition

$$
g^{\prime}: S^{3} \stackrel{\sigma}{\longrightarrow} S^{3} \vee S^{3} \stackrel{a \eta \vee \eta}{\longrightarrow} S_{1}^{2} \vee S_{2}^{2}
$$

and let $C_{g^{\prime}}$ be its cofiber. By Lemma 2.10, $\Sigma g^{\prime}$ is $\bar{\eta}_{1}$ and $p_{1} \circ g^{\prime}$ is homotopic to a $\eta$ and $p_{1} \circ g^{\prime}$ is homotopic to $a \eta$. Use Theorem 3.1 by taking $Y$ to be $S_{2}^{2}$ and $\varphi: S_{2}^{2} \rightarrow S_{1}^{2} \vee S_{2}^{2}$ and obtain $\mathcal{G}_{t}(M) \simeq \mathcal{G}_{t}\left(C_{g^{\prime}} / S_{2}^{2}\right) \times \Omega^{2} G$. Since $C_{g^{\prime}} / S_{2}^{2}$ is homotopy equivalent to $C_{a \eta}$, we have

$$
\mathcal{G}_{t}\left(C_{g^{\prime}}\right) \simeq \mathcal{G}_{t}\left(C_{a \eta}\right) \times \Omega^{2} G .
$$

Observe that $g=T \circ g^{\prime}$, where $T: S_{1}^{2} \vee S_{2}^{2} \rightarrow S_{2}^{2} \vee S_{1}^{2}$ is the swapping map. Since $T$ is a homotopy equivalence, $C_{g^{\prime}}$ and $C_{g}$ are homotopy equivalent. Combining the two homotopy equivalences gives the asserted lemma.

Proposition 2.13. Suppose that $M$ is a 4-dimensional simply-connected Poincaré-complex. Let the rank of $H^{2}(M)$ be $n$. If $\Sigma f$ is null-homotopic, then there are homotopy equivalences

$$
\Sigma M \simeq S^{5} \vee\left(\bigvee_{i=1}^{n} S^{3}\right) \quad \text { and } \quad \mathcal{G}_{t}(M) \simeq \mathcal{G}_{t}\left(S^{4}\right) \times \prod_{i=1}^{n} \Omega^{2} G
$$

If $\Sigma f$ is nontrivial, then there are homotopy equivalences

$$
\Sigma M \simeq \Sigma \mathbb{C P}^{2} \vee\left(\bigvee_{i=1}^{n-1} S^{3}\right) \text { and } \mathcal{G}_{t}(M) \simeq \mathcal{G}_{t}\left(\mathbb{C P}^{2}\right) \times \prod_{i=1}^{n-1} \Omega^{2} G
$$

Proof. If $\Sigma f$ is null-homotopic, then use Theorem 2.4 by taking $Y$ to be $M_{3}$ and $\varphi: Y \rightarrow M_{3}$ to be the identity map and get the first two homotopy equivalences.

If $\Sigma f$ is nontrivial, by Lemma 2.11 there are homotopy equivalences

$$
\Sigma M \simeq \Sigma \mathbb{C P}^{2} \vee\left(\bigvee_{i=1}^{n-1} S^{3}\right) \text { and } \mathcal{G}_{t}(M) \simeq \mathcal{G}_{t}\left(C_{a \eta}\right) \times \prod_{i=1}^{n-1} \Omega^{2} G
$$

where $a$ is an odd integer. It suffices to show that we can replace $\mathcal{G}_{t}\left(C_{a \eta}\right)$ by $\mathcal{G}_{t}\left(\mathbb{C P}^{2}\right)$.

When $n=1$, the Poincaré complex condition implies that $f$ has Hopf invariant equal to 1 or -1 . Therefore $M$ is homotopy equivalent to $\mathbb{C P}^{2}$ and the statement holds. When $n \geq 2$, there is at least one copy of $\Omega^{2} G$ on the right hand side. By Lemma 2.12, we can replace $\mathcal{G}_{t}\left(C_{a \eta}\right)$ by $\mathcal{G}_{t}\left(\mathbb{C P}^{2}\right)$ to obtain the proposition. 
It would be interesting to know if Lemma 2.12 can be improved to $\mathcal{G}_{t}\left(C_{a \eta}\right) \simeq \mathcal{G}_{t}\left(\mathbb{C P}^{2}\right)$. Observe that Proposition 2.13 is an improvement on the homotopy equivalence (1) for the non-spin case in [18], which gives a decomposition only after localization away from 2.

\section{Gauge Groups OVER NON-Simply-CONNECTED 4-MAnifoldS}

From now on we assume that $M$ is an orientable, smooth, connected, closed 4 manifold. By Morse Theory, $M$ admits a CW-structure with one 4-cell [14, Theorem 3.35]. In this section we calculate the homotopy types of $\Sigma M$ and $\mathcal{G}_{t}(M)$ when $\pi_{1}(M)$ is $(1)$ a free group $\mathbb{Z}^{* m},(2)$ a cyclic group $\mathbb{Z} / p^{r} \mathbb{Z}$, or $(3)$ a free product of types $\left(\mathbb{Z}^{* m}\right) *\left(*_{j=1}^{n} \mathbb{Z} / p_{j}^{r_{j}} \mathbb{Z}\right)$, where $p$ and the $p_{j}$ 's are odd primes. Our strategy is to apply Theorem 2.4 and its variations to decompose $\mathcal{G}_{t}(M)$ into a product of a gauge group of a simply-connected space, whose homotopy type is worked out in Proposition 2.13, and some complementary factors that do not depend on $t$.

3.1. The case when $\pi_{1}(M)=\mathbb{Z}^{* m}$. When $\pi_{1}(M)$ is a free group, $M_{3}$ is homotopy equivalent to a wedge sum of spheres [7]

$$
M_{3} \simeq\left(\bigvee_{i=1}^{m} S^{3}\right) \vee\left(\bigvee_{j=1}^{n} S^{2}\right) \vee\left(\bigvee_{k=1}^{m} S^{1}\right)
$$

Using Theorem 2.4 we can calculate the homotopy types of $\Sigma M$ and $\mathcal{G}_{t}(M)$.

Theorem 3.1. Suppose $\pi_{1}(M) \cong \mathbb{Z}^{* m}$. Let the rank of $H^{2}(M)$ be $n$. If $\Sigma f$ is null-homotopic, then there are homotopy equivalences

$$
\begin{gathered}
\Sigma M \simeq S^{5} \vee\left(\bigvee_{i=1}^{m} S^{4}\right) \vee\left(\bigvee_{j=1}^{n} S^{3}\right) \vee\left(\bigvee_{k=1}^{m} S^{2}\right) \\
\mathcal{G}_{t}(M) \simeq \mathcal{G}_{t}\left(S^{4}\right) \times \prod_{i=1}^{m} \Omega^{3} G \times \prod_{j=1}^{n} \Omega^{2} G \times \prod_{k=1}^{m} \Omega G .
\end{gathered}
$$

If $\Sigma f$ is nontrivial, then there are homotopy equivalences

$$
\begin{aligned}
\Sigma M & \simeq \Sigma \mathbb{C P}^{2} \vee\left(\bigvee_{i=1}^{m} S^{4}\right) \vee\left(\bigvee_{j=1}^{n-1} S^{3}\right) \vee\left(\bigvee_{k=1}^{m} S^{2}\right) \\
\mathcal{G}_{t}(M) & \simeq \mathcal{G}_{t}\left(\mathbb{C P}^{2}\right) \times \prod_{i=1}^{m} \Omega^{3} G \times \prod_{j=1}^{n-1} \Omega^{2} G \times \prod_{k=1}^{m} \Omega G .
\end{aligned}
$$

Proof. Denote the $i^{\text {th }}$ copy of $S^{3}$ in $M_{3}$ by $S_{i}^{3}$ and the $k^{\text {th }}$ copy of $S^{1}$ by $S_{k}^{1}$. We show that the inclusions $\varphi_{i}^{3}: S_{i}^{3} \rightarrow M_{3}$ and $\varphi_{k}^{1}: S_{k}^{1} \rightarrow M_{3}$ satisfy the hypothesis of Lemma 2.7 for all $i$ and $k$.

Let $p_{i}^{3}: M_{3} \rightarrow S_{i}^{3}$ and $p_{k}^{1}: M_{3} \rightarrow S_{k}^{1}$ be the pinch maps. Then $\Sigma p_{i}^{3}$ and $\Sigma p_{k}^{1}$ are left homotopy inverses of $\Sigma \varphi_{i}^{3}$ and $\Sigma \varphi_{k}^{1}$. Moreover, $\Sigma p_{i}^{3} \circ \Sigma f \simeq \Sigma\left(p_{i}^{3} \circ f\right)$ is null-homotopic since $f$ induces a trivial homomorphism $f^{*}: H^{3}\left(M_{3}\right) \rightarrow H^{3}\left(S^{3}\right)$, and $\Sigma p_{k}^{1} \circ \Sigma f \simeq \Sigma\left(p_{k}^{1} \circ f\right)$ is null-homotopic since $p_{k}^{1} \circ f$ is null-homotopic by $\pi_{3}\left(S^{1}\right)=0$. Apply Lemma 2.7 and get

$$
\Sigma M \simeq \Sigma M^{\prime} \vee\left(\bigvee_{i=1}^{m} S^{4}\right) \vee\left(\bigvee_{k=1}^{m} S^{2}\right) \quad \text { and } \quad \mathcal{G}_{t}(M) \simeq \mathcal{G}_{t}\left(M^{\prime}\right) \times \prod_{i=1}^{m} \Omega^{3} G \times \prod_{k=1}^{m} \Omega G
$$


where $M^{\prime}$ is the cofiber of the inclusion $\left(\bigvee_{i=1}^{m} S^{1}\right) \vee\left(\bigvee_{k=1}^{m} S^{3}\right) \hookrightarrow M$. Observe that the 3-skeleton of $M^{\prime}$ is homotopy equivalent to $\bigvee_{i=1}^{n} S^{2}$. Since $H^{2}\left(S_{i}^{1}\right)$ and $H^{2}\left(S_{k}^{3}\right)$ are zero, Lemma 2.6 implies that $M^{\prime}$ satisfies Poincaré Duality. Let $f^{\prime}$ be the attaching map of the 4-cell in $M_{3}^{\prime}$. By Proposition 2.13, if $\Sigma f^{\prime}$ is null-homotopic, then we have

$$
\Sigma M^{\prime} \simeq S^{5} \vee\left(\bigvee_{i=1}^{n} S^{3}\right) \text { and } \quad \mathcal{G}_{t}\left(M^{\prime}\right) \simeq \mathcal{G}_{t}\left(S^{4}\right) \times \prod_{i=1}^{n} \Omega^{2} G
$$

If $\Sigma f^{\prime}$ is nontrivial, then we have

$$
\Sigma M^{\prime} \simeq \Sigma \mathbb{C P}^{2} \vee\left(\bigvee_{i=1}^{n-1} S^{3}\right) \text { and } \mathcal{G}_{t}\left(M^{\prime}\right) \simeq \mathcal{G}_{t}\left(\mathbb{C P}^{2}\right) \times \prod_{i=1}^{n-1} \Omega^{2} G
$$

By Lemma $2.5 \Sigma f^{\prime}$ is null-homotopic if and only if $\Sigma f$ is null-homotopic. Combining these homotopy equivalences gives the theorem.

3.2. The case when $\pi_{1}(M)=\mathbb{Z} / p^{r} \mathbb{Z}$. Recall that an $n$-dimensional Moore space $P^{n}(k)$ is the cofiber of the degree- $k$ map $S^{n-1} \stackrel{k}{\longrightarrow} S^{n-1}$ for $n \geq 2$. With integral coefficients, $\tilde{H}_{i}\left(P^{n}(k)\right)$ is $\mathbb{Z} / k \mathbb{Z}$ for $i=n-1$ and is zero otherwise. With mod- $k$ coefficients, $\tilde{H}_{i}\left(P^{n}(k) ; \mathbb{Z} / k \mathbb{Z}\right)$ is $\mathbb{Z} / k \mathbb{Z}$ for $i=n-1$ and $n$ and is zero otherwise. Let $u$ be a generator of $\tilde{H}_{n-1}\left(P^{n}(k)\right)$. By the Universal Coefficient Theorem, $\tilde{H}_{n-1}\left(P^{n}(k) ; \mathbb{Z} / k \mathbb{Z}\right)$ is generated by the mod- $k$ reduction $\bar{u}$ of $u$, and $\tilde{H}_{n}\left(P^{n}(k) ; \mathbb{Z} / k \mathbb{Z}\right)$ is generated by $\bar{v}=\beta \bar{u}$, where $\beta$ is the Bockstein homomorphism.

For any space $X$, the mod- $k$ homotopy group $\pi_{n}(X ; \mathbb{Z} / k \mathbb{Z})$ is defined to be $\left[P^{n}(k), X\right]$. When $n \geq 3, \pi_{n}(X ; \mathbb{Z} / k \mathbb{Z})$ has a group structure induced by the comultiplication of $P^{n}(k)$ and when $n \geq 4, \pi_{n}(X ; \mathbb{Z} / k \mathbb{Z})$ is abelian. There are two associated homomorphisms: the mod- $k$ Hurewicz homomorphism

$$
\bar{h}: \pi_{n}(X ; \mathbb{Z} / k \mathbb{Z}) \rightarrow H_{n}(X ; \mathbb{Z} / k \mathbb{Z}),
$$

which is defined to be $\bar{h}(f)=f_{*}(\bar{v})$ for $f \in \pi_{n}(X ; \mathbb{Z} / k \mathbb{Z})$, and the homotopy Bockstein homomorphism

$$
\bar{\beta}_{\pi}: \pi_{n}(X ; \mathbb{Z} / k \mathbb{Z}) \rightarrow \pi_{n-1}(X),
$$

which is defined to be $\bar{\beta}_{\pi}(f)=\imath^{*} \circ f$ and $\imath: S^{n-1} \rightarrow P^{n}(k)$ is the inclusion. They are compatible with the standard Hurewicz homomorphism $h$ and Bockstein homomorphisms $\beta$ in the commutative diagram [15]

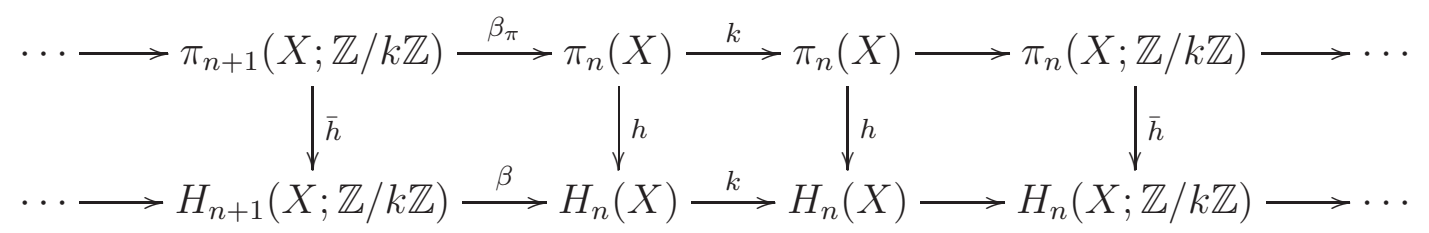

For any map $g: P^{3}(k) \rightarrow P^{3}(k)$, let $C_{g}$ be its cofiber, and let $a$ and $b$ be generators of $H^{2}\left(C_{g} ; \mathbb{Z} / k \mathbb{Z}\right)$ and $H^{4}\left(C_{g} ; \mathbb{Z} / k \mathbb{Z}\right)$. Then the mod- $k$ Hopf invariant $\bar{H}(g) \in \mathbb{Z} / k \mathbb{Z}$ is defined by the formula $a \cup a \equiv \bar{H}(g) b(\bmod k)$.

Lemma 3.2. [16, Corollary 11.12] Let $p$ be an odd prime and let $g: P^{3}\left(p^{r}\right) \rightarrow P^{3}\left(p^{r}\right)$ be a map in the kernel of $\bar{h}$. Then $g$ is null-homotopic if and only if its mod-p $p^{r}$ Hopf invariant $\bar{H}(g)$ is zero. 
Back to the calculation of $\mathcal{G}_{t}(M)$. When $\pi_{1}(M)=\mathbb{Z} / p^{r} \mathbb{Z}$, Poincaré Duality and the Universal Coefficient Theorem imply the homology groups of $M$ are as follows:

$$
H_{i}(M ; \mathbb{Z})= \begin{cases}\mathbb{Z} & i=0,4 \\ \mathbb{Z} / p^{r} \mathbb{Z} & i=1 \\ \mathbb{Z}^{\oplus n} \oplus \mathbb{Z} / p^{r} \mathbb{Z} & i=2 \\ 0 & \text { else. }\end{cases}
$$

Now we calculate the homotopy type of $\Sigma M$ to find a possible subcomplex $Y$ satisfying the hypothesis of Theorem 2.4 .

Lemma 3.3. Let $p$ be an odd prime. Then $\pi_{4}\left(P^{4}\left(p^{r}\right)\right)$ and $\pi_{4}\left(P^{3}\left(p^{r}\right)\right)$ are trivial.

Proof. After localizing away from $p$, any $P^{n}\left(p^{r}\right)$ is contractible so $\pi_{4}\left(P^{4}\left(p^{r}\right)\right)$ and $\pi_{4}\left(P^{3}\left(p^{r}\right)\right)$ are $p$-torsion. Localize at $p$ and consider the long exact sequence of homotopy groups for the pair $\left(P^{4}\left(p^{r}\right), S^{3}\right)$ :

$$
\cdots \longrightarrow \pi_{4}\left(S^{3}\right) \longrightarrow \pi_{4}\left(P^{4}\left(p^{r}\right)\right) \stackrel{j_{*}}{\longrightarrow} \pi_{4}\left(P^{4}\left(p^{r}\right), S^{3}\right) \longrightarrow \cdots
$$

The pair $\left(P^{4}\left(p^{r}\right), S^{3}\right)$ is 3-connected, so $\pi_{4}\left(P^{4}\left(p^{r}\right), S^{3}\right)$ is $\mathbb{Z}$ by Hurewicz Theorem. Since $\pi_{4}\left(S^{3}\right)$ is trivial at odd primes, $j_{*}$ is an injection. But $\pi_{4}\left(P^{4}\left(p^{r}\right)\right)$ is torsion, so this injection only makes sense if $\pi_{4}\left(P^{4}\left(p^{r}\right)\right)$ is trivial.

Now we calculate $\pi_{4}\left(P^{3}\left(p^{r}\right)\right)$. Let $F^{3}\left\{p^{r}\right\}$ be the homotopy fiber of the pinch map $P^{3}\left(p^{r}\right) \longrightarrow S^{3}$. Then $\pi_{4}\left(P^{3}\left(p^{r}\right)\right)$ equals $\pi_{4}\left(F^{3}\left\{p^{r}\right\}\right)$ since $\pi_{4}\left(\Omega S^{3}\right)$ and $\pi_{4}\left(S^{3}\right)$ are trivial at odd primes. By [15, Proposition 11.7.1], there is a $p$-local homotopy equivalence

$$
\Omega F^{3}\left\{p^{r}\right\} \simeq S^{1} \times \Omega \Sigma\left(\bigvee_{\alpha} P^{n_{\alpha}}\left(p^{r}\right)\right) \times \prod_{j} S^{2 p^{j}-1}\left\{p^{r+1}\right\},
$$

where $n_{\alpha}$ is either 3 or greater than 4 , and $S^{n}\left\{p^{r+1}\right\}$ is the homotopy fiber of the degree map $p^{r+1}: S^{n} \longrightarrow S^{n}$. Since $\pi_{4}\left(F^{3}\left\{p^{r}\right\}\right)$ is $\pi_{3}\left(\Omega F^{3}\left\{p^{r}\right\}\right)$, we need to calculate the third homotopy group of each factor on the right hand side. The first factor $\pi_{3}\left(S^{1}\right)$ and the last factor is trivial since $S^{2 p^{j}-1}\left\{p^{r+1}\right\}$ is 3-connected for $j \geq 1$. For the remaining factor of $\Omega F^{3}\left\{p^{r}\right\}$, consider the string of isomorphisms

$$
\begin{aligned}
\pi_{3}\left(\Omega \Sigma\left(\bigvee_{\alpha} P^{n_{\alpha}}\left(p^{r}\right)\right)\right) & \cong \pi_{3}\left(\prod_{\alpha} \Omega \Sigma P^{n_{\alpha}}\left(p^{r}\right)\right) \\
& \cong \pi_{3}\left(\Omega \Sigma P^{3}\left(p^{r}\right)\right) \\
& \cong \pi_{4}\left(P^{4}\left(p^{r}\right)\right) \\
& \cong 0 .
\end{aligned}
$$

The first isomorphism is obtained from the Hilton-Milnor Theorem using dimension and connectivity considerations. The second isomorphism holds since only one $n_{\alpha}$ equals 3 while the rest are strictly larger than 4 . The third isomorphism holds by adjunction, and the fourth isomorphism holds as we have already seen that $\pi_{4}\left(P^{4}\left(p^{r}\right)\right)=0$. Therefore $\pi_{4}\left(F^{3}\left\{p^{r}\right\}\right)$ is trivial and so is $\pi_{4}\left(P^{3}\left(p^{r}\right)\right)$.

Lemma 3.4. If $\pi_{1}(M) \cong \mathbb{Z} / p^{r} \mathbb{Z}$, then there is a homotopy equivalence

$$
\Sigma M_{3} \simeq P^{4}\left(p^{r}\right) \vee\left(\bigvee_{i=1}^{n} S^{3}\right) \vee P^{3}\left(p^{r}\right)
$$


Proof. Since $\Sigma M_{3}$ is simply-connected, it has a minimal cell structure. By [6, Proposition 4H.3] $\Sigma M_{3}$ is homotopy equivalent to the cofiber of a map

$$
g: P^{3}\left(p^{r}\right) \vee\left(\bigvee_{i=1}^{n} S^{2}\right) \rightarrow P^{3}\left(p^{r}\right)
$$

such that $g$ induces a trivial homomorphism $g_{*}: H_{2}\left(P^{3}\left(p^{r}\right) \vee\left(\bigvee_{i=1}^{n} S^{2}\right)\right) \rightarrow H_{2}\left(P^{3}\left(p^{r}\right)\right)$. We claim that $g$ is null-homotopic. To distinguish the 2 -spheres in the wedge sum, denote the $i^{\text {th }}$ copy by $S_{i}^{2}$. Since

$$
\left[P^{3}\left(p^{r}\right) \vee\left(\bigvee_{i=1}^{n} S_{i}^{2}\right), P^{3}\left(p^{r}\right)\right] \cong\left[P^{3}\left(p^{r}\right), P^{3}\left(p^{r}\right)\right] \oplus\left(\bigoplus_{i=1}^{n}\left[S_{i}^{2}, P^{3}\left(p^{r}\right)\right]\right)
$$

we write $g=g^{\prime} \oplus\left(\bigoplus_{i=1}^{n} g_{i}^{\prime \prime}\right)$, where $g^{\prime} \in\left[P^{3}\left(p^{r}\right), P^{3}\left(p^{r}\right)\right]$ and $g_{i}^{\prime \prime} \in\left[S_{i}^{2}, P^{3}\left(p^{r}\right)\right]$.

Consider the commutative diagram

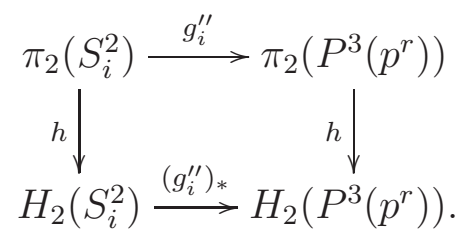

Both Hurewicz homomorphisms $h$ are isomorphisms by Hurewicz Theorem. Since $g_{*}$ is trivial, so is $\left(g_{i}^{\prime \prime}\right)_{*}$. The diagram implies that $g_{i}^{\prime \prime}$ is null-homotopic. Therefore $\Sigma M_{3}$ is homotopy equivalent to $C_{g^{\prime}} \vee\left(\bigvee_{i=1}^{n} S^{3}\right)$, where $C_{g^{\prime}}$ is the cofiber of $g^{\prime}$. It suffices to show that $g^{\prime}$ is null-homotopic.

Consider the commutative diagram

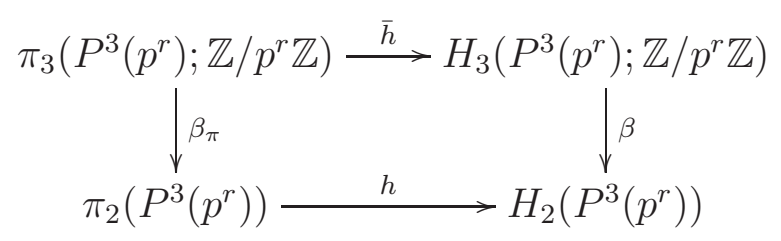

from (6). The induced homomorphism $g_{*}^{\prime}$ is trivial and we have

$$
h \circ \beta_{\pi}\left(g^{\prime}\right)=\beta \circ \bar{h}\left(g^{\prime}\right)=\beta \circ\left(\left(g^{\prime}\right)_{*} v\right)=0 .
$$

Observe that $\beta: H_{3}\left(P^{3}\left(p^{r}\right) ; \mathbb{Z} / p^{r} \mathbb{Z}\right) \rightarrow H_{2}\left(P^{3}\left(p^{r}\right)\right)$ is an isomorphism in this case, so $g^{\prime}$ is in the kernel of $\bar{h}$. Since $C_{g^{\prime}}$ retracts off the suspension $\Sigma M_{3}$, it is a co-H-space and $H^{*}\left(C_{g^{\prime}} ; \mathbb{Z} / p^{r} \mathbb{Z}\right)$ has trivial cup products. Therefore the mod- $p^{r}$ Hopf invariant $\bar{H}\left(g^{\prime}\right)$ is zero and $g^{\prime}$ is nullhomotopic by Lemma 3.2.

Lemma 3.4 says that $\Sigma M_{3}$ contains $P^{3}\left(p^{r}\right) \vee P^{4}\left(p^{r}\right)$ as its wedge summands. This, however, does not necessarily imply that $M_{3}$ contains $P^{2}\left(p^{r}\right) \vee P^{3}\left(p^{r}\right)$ since $M$ is not simply-connected.

Lemma 3.5. If $\pi_{1}(M) \cong \mathbb{Z} / p^{r} \mathbb{Z}$, then there exists a map $\epsilon: P^{2}\left(p^{r}\right) \rightarrow M_{3}$ satisfying the hypothesis of Theorem 2.4 and its cofiber $C_{\epsilon}$ is simply-connected. 
Proof. By the Cellular Approximation Theorem, $\pi_{1}\left(M_{3}\right)$ equals $\pi_{1}(M) \cong \mathbb{Z} / p^{r} \mathbb{Z}$. Let $j: S^{1} \rightarrow M_{3}$ represent a generator of $\pi_{1}\left(M_{3}\right)$. It has order $p^{r}$, so there exists an extension $\epsilon: P^{2}\left(p^{r}\right) \rightarrow M_{3}$.

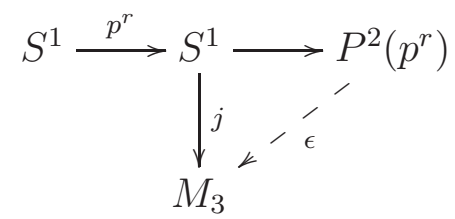

Since $\pi_{1}\left(M_{3}\right)$ is abelian, $H_{1}\left(M_{3}\right)$ is $\pi_{1}\left(M_{3}\right) \cong \mathbb{Z} / p^{r} \mathbb{Z}$ by Hurewicz Theorem and the induced map $\epsilon_{*}: H_{1}\left(P^{2}\left(p^{r}\right)\right) \rightarrow H_{1}\left(M_{3}\right)$ is an isomorphism. Therefore the cofiber $C_{\epsilon}$ of $\epsilon$ has $H_{1}\left(C_{\epsilon}\right)=0$, implying that $C_{\epsilon}$ is simply-connected.

Now we show that $\Sigma \epsilon$ has a left homotopy inverse. Let $\psi$ be the composition

$$
\psi: \Sigma M_{3} \simeq P^{4}\left(p^{r}\right) \vee\left(\bigvee_{i=1}^{n} S_{i}^{3}\right) \vee P^{3}\left(p^{r}\right) \stackrel{\text { pinch }}{\longrightarrow} P^{3}\left(p^{r}\right)
$$

Observe that

$$
\psi_{*}: H_{2}\left(\Sigma M_{3} ; \mathbb{Z} / p^{r} \mathbb{Z}\right) \rightarrow H_{2}\left(P^{3}\left(p^{r}\right) ; \mathbb{Z} / p^{r} \mathbb{Z}\right) \text { and }(\Sigma \epsilon)_{*}: H_{2}\left(P^{3}\left(p^{r}\right) ; \mathbb{Z} / p^{r} \mathbb{Z}\right) \rightarrow H_{2}\left(\Sigma M_{3} ; \mathbb{Z} / p^{r} \mathbb{Z}\right)
$$

are isomorphism, so $(\psi \circ \Sigma \epsilon)_{*}: H_{2}\left(P^{3}\left(p^{r}\right) ; \mathbb{Z} / p^{r} \mathbb{Z}\right) \rightarrow H_{2}\left(P^{3}\left(p^{r}\right) ; \mathbb{Z} / p^{r} \mathbb{Z}\right)$ is an isomorphism. Then Bockstein homomorphism implies $(\psi \circ \Sigma \epsilon)_{*}: H_{3}\left(P^{3}\left(p^{r}\right) ; \mathbb{Z} / p^{r} \mathbb{Z}\right) \rightarrow H_{3}\left(P^{3}\left(p^{r}\right) ; \mathbb{Z} / p^{r} \mathbb{Z}\right)$ is an isomorphism. Therefore $\psi \circ \Sigma \epsilon$ is a homotopy equivalence and $\psi$ is a left homotopy inverse of $\Sigma \epsilon$.

Moreover, the composition $\psi \circ \Sigma f$ is null-homotopic since $\pi_{4}\left(P^{3}\left(p^{r}\right)\right)$ is trivial by Lemma 3.3. Therefore $\epsilon$ satisfies the hypothesis of Theorem 2.4.

Denote the pointed mapping space $\operatorname{Map}^{*}\left(P^{n}\left(p^{r}\right), G\right)$ by $\Omega^{n} G\left\{p^{r}\right\}$. This notation is justified since $\operatorname{Map}^{*}\left(P^{n}\left(p^{r}\right), G\right)$ is the homotopy fiber of the power map $p^{r}: \Omega^{n} G \rightarrow \Omega^{n} G$. Now we calculate the homotopy type of $\mathcal{G}_{t}(M)$.

Theorem 3.6. Suppose $\pi_{1}(M) \cong \mathbb{Z} / p^{r} \mathbb{Z}$ where $p$ is an odd prime. Let the rank of $H^{2}(M)$ be $n$. If $\Sigma f$ is null-homotopic, then there are homotopy equivalences

$$
\begin{gathered}
\Sigma M \simeq S^{5} \vee P^{4}\left(p^{r}\right) \vee\left(\bigvee_{i=1}^{n} S^{3}\right) \vee P^{3}\left(p^{r}\right) \\
\mathcal{G}_{t}(M) \simeq \mathcal{G}_{t}\left(S^{4}\right) \times \Omega^{3} G\left\{p^{r}\right\} \times \prod_{i=1}^{n} \Omega^{2} G \times \Omega^{2} G\left\{p^{r}\right\} .
\end{gathered}
$$

If $\Sigma f$ is nontrivial, then there are homotopy equivalences

$$
\begin{gathered}
\Sigma M \simeq \Sigma \mathbb{C P}^{2} \vee P^{4}\left(p^{r}\right) \vee\left(\bigvee_{i=1}^{n-1} S^{3}\right) \vee P^{3}\left(p^{r}\right) \\
\mathcal{G}_{t}(M) \simeq \mathcal{G}_{t}\left(\mathbb{C P}^{2}\right) \times \Omega^{3} G\left\{p^{r}\right\} \times \prod_{i=1}^{n-1} \Omega^{2} G \times \Omega^{2} G\left\{p^{r}\right\} .
\end{gathered}
$$

Proof. We decompose $\mathcal{G}_{t}(M)$ in the following steps.

Step 1: By Lemma 3.5, there exists a map $\epsilon: P^{2}\left(p^{r}\right) \rightarrow M_{3}$ satisfying the hypothesis of Theorem 2.4. Apply the theorem and get

$$
\Sigma M \simeq \Sigma M^{\prime} \vee P^{3}\left(p^{r}\right) \text { and } \mathcal{G}_{t}(M) \simeq \mathcal{G}_{t}\left(M^{\prime}\right) \times \Omega^{2} G\left\{p^{r}\right\} .
$$


where $M^{\prime}$ is the cofiber of $P^{2}\left(p^{r}\right) \stackrel{\epsilon}{\longrightarrow} M_{3} \hookrightarrow M$.

Step 2: By Lemma 3.5 $M^{\prime}$ is simply-connected, so its 3-skeleton $M_{3}^{\prime}$ is $\left(\bigvee_{i=1}^{n}\right) S^{2} \vee P^{3}\left(p^{r}\right)$. We show that the inclusion $\varphi: P^{3}\left(p^{r}\right) \hookrightarrow M_{3}^{\prime}$ satisfies the condition of Theorem 2.4. The pinch map $\Sigma M_{3}^{\prime} \rightarrow P^{4}\left(p^{r}\right)$ is a left homotopy inverse of $\Sigma \varphi$. Let $f^{\prime}$ be the attaching map of the 4-cell in $M^{\prime}$. Then the composition

$$
S^{4} \stackrel{\Sigma f^{\prime}}{\longrightarrow} \Sigma M_{3}^{\prime} \stackrel{\text { pinch }}{\longrightarrow} P^{4}\left(p^{r}\right)
$$

is null-homotopic since $\pi_{4}\left(P^{4}\left(p^{r}\right)\right)$ is trivial by Lemma 3.3. Apply Theorem 2.4 and get

$$
\Sigma M^{\prime} \simeq \Sigma M^{\prime \prime} \vee P^{4}\left(p^{r}\right) \text { and } \mathcal{G}_{t}\left(M^{\prime}\right) \simeq \mathcal{G}_{t}\left(M^{\prime \prime}\right) \times \Omega^{3} G\left\{p^{r}\right\}
$$

where $M^{\prime \prime}$ is $M^{\prime} / P^{3}\left(p^{r}\right)$.

Step 3: By Lemma 3.4 the 3-skeleton of $M^{\prime \prime}$ is homotopy equivalent to $\bigvee_{i=1}^{n} S^{2}$. Since $H^{2}\left(P^{2}\left(p^{r}\right)\right)$ and $H^{2}\left(P^{3}\left(p^{r}\right)\right)$ are torsion, Lemma 2.6 implies that $M^{\prime \prime}$ satisfies Poincaré Duality. Let $f^{\prime \prime}$ be the attaching map of the 4-cell in $M^{\prime \prime}$. By Proposition 2.13, if $\Sigma f^{\prime \prime}$ is null-homotopic, then

$$
\Sigma M^{\prime \prime} \simeq S^{5} \vee\left(\bigvee_{i=1}^{n} S^{3}\right) \text { and } \mathcal{G}_{t}\left(M^{\prime \prime}\right) \simeq \mathcal{G}_{t}\left(S^{4}\right) \times \prod_{i=1}^{n} \Omega^{2} G
$$

If $\Sigma f^{\prime \prime}$ is nontrivial, then

$$
\Sigma M^{\prime \prime} \simeq \Sigma \mathbb{C P}^{2} \vee\left(\bigvee_{i=1}^{n-1} S^{3}\right) \text { and } \mathcal{G}_{t}\left(M^{\prime \prime}\right) \simeq \mathcal{G}_{t}\left(\mathbb{C P}^{2}\right) \times \prod_{i=1}^{n-1} \Omega^{2} G
$$

Step 4: Combining all the homotopy equivalences from Step 1 to 3 gives the theorem.

3.3. The case when $\pi_{1}=\left(\mathbb{Z}^{* m}\right) *\left(*_{j=1}^{n} \mathbb{Z} / p_{j}^{r_{j}} \mathbb{Z}\right)$. Suppose that $\pi_{1}(M)$ is $\left(\mathbb{Z}^{* m}\right) *\left(*_{j=1}^{n} \mathbb{Z} / p_{j}^{r_{j}} \mathbb{Z}\right)$, where $p_{j}$ is an odd prime. The Stable Decomposition Theorem [13, Theorem 1.3] implies that for some number $d$ there is a diffeomorphism

$$
M \#_{d}\left(S^{2} \times S^{2}\right) \cong N \#\left(\#_{j=1}^{n} L_{j}\right),
$$

where $N$ and $L_{j}$ 's are orientable, smooth, connected, closed 4-manifolds with $\pi_{1}(N)=\mathbb{Z}^{* m}$ and $\pi_{1}\left(L_{j}\right)=\mathbb{Z} / p_{j}^{r_{j}} \mathbb{Z}$. We will calculate the suspensions and gauge groups for both sides of isomorphism (7).

Lemma 3.7. There are homotopy equivalences

$$
\Sigma\left(M \#_{d}\left(S^{2} \times S^{2}\right)\right) \simeq(\Sigma M) \vee\left(\bigvee_{s=1}^{2 d} S^{3}\right) \quad \text { and } \quad \mathcal{G}_{t}\left(M \#_{d}\left(S^{2} \times S^{2}\right)\right) \simeq \mathcal{G}_{t}(M) \times \prod_{s=1}^{2 d} \Omega^{2} G
$$

Proof. By induction it suffices to show the lemma for the $d=1$ case. We use Lemma 2.8 to prove it. For $M$, the inclusion $\varphi:\left\{m_{0}\right\} \hookrightarrow M$ of the basepoint $m_{0}$ obviously satisfies the hypothesis of Theorem 2.4. For $S^{2} \times S^{2}$, take the inclusion $\varphi^{\prime}: S^{2} \vee S^{2} \hookrightarrow S^{2} \times S^{2}$ to be the inclusion of the 3-skeleton. Since $\Sigma\left(S^{2} \times S^{2}\right)$ is homotopy equivalent to $S^{5} \vee S^{3} \vee S^{3}, \varphi^{\prime}$ satisfies the hypothesis as well. Apply Lemma 2.8 to get

$$
\Sigma\left(M \#\left(S^{2} \times S^{2}\right)\right) \simeq\left(\Sigma C_{j}\right) \vee\left(\bigvee_{s=1}^{2} S^{3}\right) \text { and } \mathcal{G}_{t}\left(M \#\left(S^{2} \times S^{2}\right)\right) \simeq \mathcal{G}_{t}\left(C_{j}\right) \times \prod_{s=1}^{2} \Omega^{2} G
$$


where $C_{j}$ is the cofiber of the inclusion $j:\left\{m_{0}\right\} \vee\left(S^{2} \vee S^{2}\right) \hookrightarrow M \#\left(S^{2} \times S^{2}\right)$. Consider the cofibration diagram

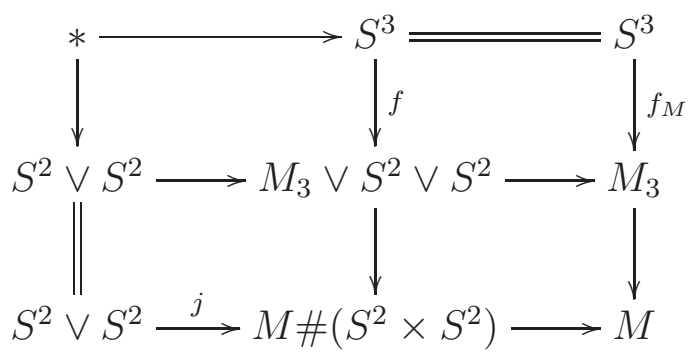

where $f_{M}$ is the attaching map of the 4-cell in $M$. The bottom row implies that $C_{j}$ is homotopy equivalent to $M$ and the asserted homotopy equivalences follow.

Theorem 3.8. Let $\pi_{1}(M) \cong\left(\mathbb{Z}^{* m}\right) *\left(*_{j=1}^{n} \mathbb{Z} / p_{j}^{r_{j}} \mathbb{Z}\right)$ where $p_{j}$ is an odd prime and let $l$ be the rank of $H^{2}(M)$. Then there exists a number d such that $\Sigma\left(M \#_{d}\left(S^{2} \times S^{2}\right)\right)$ is homotopy equivalent to either

$$
\begin{aligned}
& S^{5} \vee\left(\bigvee_{i=1}^{m} S^{4}\right) \vee\left(\bigvee_{j=1}^{n} P^{4}\left(p_{j}^{r_{j}}\right)\right) \vee\left(\bigvee_{k=1}^{l+2 d} S^{3}\right) \vee\left(\bigvee_{j^{\prime}=1}^{n} P^{3}\left(p_{j^{\prime}}^{r_{j^{\prime}}}\right)\right) \vee\left(\bigvee_{i^{\prime}=1}^{m} S^{2}\right) \text { or } \\
& \Sigma \mathbb{C P}^{2} \vee\left(\bigvee_{i=1}^{m} S^{4}\right) \vee\left(\bigvee_{j=1}^{n} P^{4}\left(p_{j}^{r_{j}}\right)\right) \vee\left(\bigvee_{k=1}^{l+2 d-1} S^{3}\right) \vee\left(\bigvee_{j^{\prime}=1}^{n} P^{3}\left(p_{j^{\prime}}^{r_{j^{\prime}}}\right)\right) \vee\left(\bigvee_{i^{\prime}=1}^{m} S^{2}\right) .
\end{aligned}
$$

In the first case, we have

$\mathcal{G}_{t}(M) \times \prod_{s=1}^{2 d} \Omega^{2} G \simeq \mathcal{G}_{t}\left(S^{4}\right) \times \prod_{i=1}^{m} \Omega^{3} G \times \prod_{j=1}^{n} \Omega^{3} G\left\{p_{j}^{r_{j}}\right\} \times \prod_{k=1}^{l+2 d} \Omega^{2} G \times \prod_{j^{\prime}=1}^{n} \Omega^{2} G\left\{p_{j^{\prime}}^{r_{j^{\prime}}}\right\} \times \prod_{i^{\prime}=1}^{m} \Omega G$.

In the second case, we have

$\mathcal{G}_{t}(M) \times \prod_{s=1}^{2 d} \Omega^{2} G \simeq \mathcal{G}_{t}\left(\mathbb{C P}^{2}\right) \times \prod_{i=1}^{m} \Omega^{3} G \times \prod_{j=1}^{n} \Omega^{3} G\left\{p_{j}^{r_{j}}\right\} \times \prod_{k=1}^{l+2 d-1} \Omega^{2} G \times \prod_{j^{\prime}=1}^{n} \Omega^{2} G\left\{p_{j^{\prime}}^{r_{j^{\prime}}}\right\} \times \prod_{i^{\prime}=1}^{m} \Omega G$.

Proof. Denote $N \#\left(\#_{j=1}^{n} L_{j}\right)$ by $X$. Using the stable decomposition (7) and Lemma 3.7, we only need to calculate $\Sigma X$ and $\mathcal{G}_{t}(X)$. Let $N_{3}$ and $\left(L_{j}\right)_{3}$ be the 3 -skeletons of $N$ and $L_{j}$. Then the 3-skeleton $X_{3}$ of $X$ is the wedge sum $N_{3} \vee\left(\bigvee_{j=1}^{n}\left(L_{j}\right)_{3}\right)$.

Step 1: The 3-skeleton $N_{3}$ is homotopy equivalent to $\left(\bigvee_{i=1}^{m} S^{3}\right) \vee\left(\bigvee_{j^{\prime}=1}^{l^{\prime}} S^{2}\right) \vee\left(\bigvee_{k=1}^{m} S^{1}\right)$. Denote the $i^{\text {th }}$ copy of $S^{3}$ in $N_{3}$ by $S_{i}^{3}$ and the $k^{\text {th }}$ copy of $S^{1}$ by $S_{k}^{1}$. In the proofs of Theorem 3.1, we show that inclusions $\varphi_{i}^{3}: S_{i}^{3} \rightarrow N_{3}$ and $\varphi_{k}^{1}: S_{k}^{1} \rightarrow N_{3}$ satisfy the hypothesis of Theorem 2.4. For each $j$, by Lemma 3.5 there exists a map $\epsilon_{j}: P^{2}\left(p_{j}^{r_{j}}\right) \rightarrow\left(L_{j}\right)_{3}$ satisfying the hypothesis as well. Apply Lemma 2.8 and get

$$
\begin{aligned}
& \Sigma X \simeq \Sigma X^{\prime} \vee\left(\bigvee_{i=1}^{m} S^{4}\right) \vee\left(\bigvee_{k=1}^{m} S^{2}\right) \vee\left(\bigvee_{j=1}^{n} P^{3}\left(p_{j}^{r_{j}}\right)\right) \\
& \mathcal{G}_{t}(X) \simeq \mathcal{G}_{t}\left(X^{\prime}\right) \times \prod_{i=1}^{m} \Omega^{3} G \times \prod_{k=1}^{m} \Omega G \times \prod_{j=1}^{n} \Omega G\left\{p_{j}^{r_{j}}\right\}
\end{aligned}
$$

where $X^{\prime}$ is the cofiber of the inclusion $\left(\bigvee_{i=1}^{m} S^{3}\right) \vee\left(\bigvee_{k=1}^{m} S^{1}\right) \vee\left(\bigvee_{j=1}^{n} P^{2}\left(p_{j}^{r_{j}}\right)\right) \hookrightarrow X$. 
Step 2: Since $X^{\prime}$ is simply-connected, its 3 -skeleton $X_{3}^{\prime}$ has a minimal cell structure

$$
X_{3} \simeq\left(\bigvee_{j^{\prime}=1}^{l^{\prime \prime}} S^{2}\right) \vee\left(\bigvee_{j=1}^{n} P^{3}\left(p_{j}^{r_{j}}\right)\right)
$$

We show that inclusions $\varphi_{j}: P^{3}\left(p_{j}^{r_{j}}\right) \rightarrow X_{3}^{\prime}$ satisfy the hypothesis of Theorem 2.4. For each $j$, the pinch maps $\Sigma X_{3}^{\prime} \rightarrow P^{4}\left(p_{j}^{r_{j}}\right)$ is a left homotopy inverse of $\Sigma \varphi_{j}$. Let $f^{\prime}$ be the attaching map of the 4-cell in $X^{\prime}$. Then the composition

$$
S^{4} \stackrel{\Sigma f^{\prime}}{\longrightarrow} \Sigma X_{3}^{\prime} \stackrel{\text { pinch }}{\longrightarrow} P^{4}\left(p_{j}^{r_{j}}\right)
$$

is null-homotopic since $\pi_{4}\left(P^{3}\left(p_{j}^{r_{j}}\right)\right)$ is trivial by Lemma 3.3. Apply Lemma 2.7 and get

$$
\Sigma X^{\prime} \simeq \Sigma X^{\prime \prime} \vee\left(\bigvee_{j=1}^{n} P^{3}\left(p_{j}^{r_{j}}\right)\right) \quad \text { and } \quad \mathcal{G}_{t}\left(X^{\prime}\right) \simeq \mathcal{G}_{t}\left(X^{\prime \prime}\right) \times \prod_{j=1}^{n} \Omega^{2} G\left\{p_{j}^{r_{j}}\right\}
$$

where $X^{\prime \prime}$ is $X^{\prime} /\left(\bigvee_{j=1}^{n} P^{3}\left(p_{j}^{r_{j}}\right)\right)$.

Step 3: The 3-skeleton of $X^{\prime \prime}$ is homotopy equivalent to $\bigvee_{j^{\prime}=1}^{l^{\prime \prime}} S^{2}$. Since the subcomplexes $S_{i}^{1}, S_{k}^{3}, P^{2}\left(p_{j}^{r_{j}}\right)$ and $P^{3}\left(p_{j}^{r_{j}}\right)$ in Step 1 and 2 have either zero or torsion second cohomology groups, Lemma 2.6 implies that $X^{\prime \prime}$ satisfies Poincaré Duality. By Proposition 2.13, we have

$$
\Sigma X^{\prime \prime} \simeq S^{5} \vee\left(\bigvee_{j^{\prime}=1}^{l^{\prime \prime}} S^{3}\right) \text { and } \mathcal{G}_{t}\left(X^{\prime \prime}\right) \simeq \mathcal{G}_{t}\left(S^{4}\right) \times \prod_{j^{\prime}=1}^{l^{\prime \prime}} \Omega^{2} G
$$

or

$$
\Sigma X^{\prime \prime} \simeq \Sigma \mathbb{C P}^{2} \vee\left(\bigvee_{j^{\prime}=1}^{l^{\prime \prime}-1} S^{3}\right) \text { and } \mathcal{G}_{t}\left(X^{\prime \prime}\right) \simeq \mathcal{G}_{t}\left(\mathbb{C P}^{2}\right) \times \prod_{j^{\prime}=1}^{l^{\prime \prime}-1} \Omega^{2} G .
$$

Step 4: Combining all homotopy equivalences from Step 1 to 3, the stable decomposition (7) and Lemma 3.7 together imply the theorem. Furthermore, $H_{2}\left(M \#_{d}\left(S^{2} \times S^{2}\right)\right)$ has rank $l=2 d$, so $l^{\prime \prime}=l+2 d$.

Remark: The proofs of Theorem 3.1 and 3.6 are also valid for any orientable 4-dimensional CW-complex with one 4-cell, while Theorem 3.8 requires the smoothness of $M$ for the stable diffeomorphism splitting in [13]. One can ask under what condition the stabilizing factor $\#_{d}\left(S^{2} \times S^{2}\right)$ can be cancelled so that the factors $\prod_{s=1}^{2 d} \Omega^{2} G$ can be removed from the equation. If this can be achieved, Theorem 3.1 and 3.6 will be corollaries of Theorem 3.8 when $M$ is smooth.

\section{REFERENCES}

[1] M. Atiyah and R. Bott, The Yang-Mills equations over Riemann surfaces, Philosophical Transactions of the Royal Society of London. Series A, Mathematical and Physical Sciences, 308(1505), (1983), 523 -615 .

[2] F. Cohen, J. Moore, J. Neisendorfer, Torsion in homotopy groups, Annals of Mathematics, Second Series, 109, (1979), $121-168$.

[3] H. Hamanaka and A. Kono, Unstable K-group and homotopy type of certain gauge groups, Proceedings of the Royal Society of Edinburgh Section A: Mathematics, 136, (2006), 149 - 155.

[4] D. Gottlieb, Applications of bundle map theory, Transaction of the American Mathematical Society, 171, (1972), 23 - 50. 
[5] M. Greenberg and J. Harper, Algebraic Topology: A First Course, Benjamin/Cummings Publishing Company, 1981.

[6] A. Hatcher, Algebraic Topology, Cambridge University Press, 2002.

[7] T. Matumoto and A. Katanaga, On 4-dimensional closed manifolds with free fundamental groups, Hiroshima Math J, 25, (1995), 367 - 370.

[8] D. Kishimoto, A. Kono, On the homotopy types of $S p(n)$ gauge groups, arXiv:1803.06477.

[9] D. Kishimoto, A. Kono, M. Tsutaya, On p-local homotopy types of gauge groups, Proceedings of the Royal Society of Edinburgh Section A: Mathematics, 144, (2014), 149 - 160.

[10] D. Kishimoto, S. Theriault, M. Tsutaya, The homotopy types of $G_{2}$-gauge groups, Topology and its Applications, 228, (2017), 92 - 107.

[11] A. Kono, A note on the homotopy type of certain gauge groups, Proceedings of the Royal Society of Edinburgh Section A: Mathematics, 117, (1991), 295 - 297.

[12] A. Kono and S. Tsukuda, A remark on the homotopy type of certain gauge groups, Journal of Mathematics of Kyoto University, 36, (1996), 115 - 121.

[13] M. Kreck, W. Lück and P. Teichner, Stable prime decompositions of 4-manifolds Prospects in Topology edited by F. Quinn, Annuals of Mathematics Studies, 138, Princeton University Press, Princeton, (1995), $251-269$.

[14] Y. Matsumoto, An Introduction to Morse Theory (Translations of Mathematical Monographs), American Mathematical Society, 2002.

[15] J. Neisendorfer, Algebraic Methods in Unstable Homotopy Theory, Cambridge University Press, 2010.

[16] J. Neisendorfer, Homotopy groups with coefficients, Journal of Fixed Point Theory and Applications, 8, (2010), $247-338$.

[17] W. Sutherland, Function spaces related to gauge groups, Proceedings of the Royal Society of Edinburgh, 121A, (1992), $185-190$.

[18] S. Theriault, Odd primary homotopy decompositions of gauge groups, Algebraic and Geometric Topology, 10, (2010), $535-564$.

[19] S. Theriault, The homotopy types of Sp(2)-gauge groups, Kyoto Journal of Mathematics, 50, (2010), $591-605$.

[20] S. Theriault, Homotopy types of SU(3)-gauge groups, Publ. RIMS Kyoto Univ., 48, (2012), 543 - 563.

[21] S. Theriault, The homotopy types of SU(5)-gauge groups, Osaka Journal of Mathematics, 52, (2015), $15-31$.

[22] S. Theriault, Odd primary homotopy types of $S U(n)$-gauge groups, Algebraic and Geometric Topology, 17, (2017), 1131 - 1150.

Mathematical Sciences, University of Southampton, SO17 1BJ, UK

E-mail address: tls1g14@soton.ac.uk 